\title{
The DarTG toxin-antitoxin system provides phage defense by ADP-ribosylating viral DNA
}

Michele LeRoux ${ }^{1}$, Sriram Srikant ${ }^{1}$, Megan H. Littlehale ${ }^{1}$, Gabriella Teodoro ${ }^{1}$, Shany Doron ${ }^{2}$, Mohsen Badiee $^{3}$, Anthony K. L. Leung ${ }^{3}$, Rotem Sorek ${ }^{2}$, Michael T. Laub ${ }^{1,4,5}$

${ }^{1}$ Department of Biology, Massachusetts Institute of Technology, Cambridge, MA 02139, USA

${ }^{2}$ Department of Molecular Genetics, Weizmann Institute of Science, Rehovot, Israel

${ }^{3}$ Department of Molecular Biology and Genetics, Department of Genetic Medicine, Department of Oncology, School of Medicine, Johns Hopkins University, Baltimore, MD 21205, USA

${ }^{4}$ Howard Hughes Medical Institute, Massachusetts Institute of Technology, Cambridge, MA 02139, USA

${ }^{5}$ Correspondence can be addressed to MTL: laub@mit.edu, 617-324-0418 


\begin{abstract}
Toxin-antitoxin (TA) systems are broadly distributed, yet poorly conserved, genetic elements whose biological functions are unclear and controversial. Some TA systems may provide bacteria with immunity to infection by their ubiquitous viral predators, the bacteriophage. To identify TA systems that protect E. coli MG1655 against phage, we searched for those frequently encoded near known phage defense genes in bacterial genomes. Two of the systems tested provide strong protection against phage infection and are homologs of DarTG, a recently discovered family of TA systems whose biological functions and natural activating conditions were unclear. We demonstrate that phage infection triggers the release of DarT toxin, a DNA ADP-ribosyltransferase, to modify viral DNA and prevent replication, thereby blocking the production of mature virions. Phages can evolve to overcome DarTG defense either through mutations to their DNA polymerase or to an anti-DarT factor, gp61.2, encoded by many T-even phages. Collectively, our results indicate that phage defense may be a common function for TA systems and reveal the mechanism by which DarTG systems inhibit phage infection.
\end{abstract}




\section{Introduction}

Bacteriophage, or phage, are the nearly ubiquitous viruses that infect bacteria. Their co-evolution with bacteria has led to an abundance of bacterial defense mechanisms. CRISPR and restrictionmodification systems are two well-known phage defense systems that have been extensively characterized and famously co-opted as indispensable tools for molecular biology. In recent years, due to a recognition of the vast, unexplored biological potential of such systems, and the renewed interest in phage therapy as an alternative to antibiotics, there has been an explosion in the number of newly identified phage defense systems identified (Bernheim et al., 2020; Cohen et al., 2019; Doron et al., 2018; Gao et al., 2020). However, in most cases, the mechanism of action of these phage defense systems remain unknown or incompletely elucidated.

One class of genetic elements increasingly implicated in phage defense are toxin-antitoxin (TA) systems, which are found in nearly all sequenced bacterial chromosomes, with some species encoding dozens of different systems (Harms et al., 2018; Song and Wood, 2020). These systems typically feature a two-gene operon that encodes a growth-inhibiting toxin and a cognate, neutralizing antitoxin, which is often less stable than the toxin (Harms et al., 2018). TA systems are categorized based on the nature of the antitoxin, which may consist of a small non-coding RNA that prevents toxin translation (type I), a protein that directly interacts with and neutralizes the toxin (type II), a non-coding RNA that directly interacts with the toxin (type III), or a protein that enzymatically reverses the activity of the toxin (type IV) (Harms et al., 2018).

The biological functions of chromosomally-encoded TA systems have remained elusive and controversial (Harms et al., 2018; Page and Peti, 2016; Ronneau and Helaine, 2019; Yamaguchi and Inouye, 2011). Cells expend significant resources in keeping these systems in an "off" state, in which toxin is neutralized by antitoxin (LeRoux et al., 2020). Despite the seemingly high cost of their maintenance, the prevalence of TA systems suggests they are important for bacterial survival. TA systems are often postulated to be stress-response elements (Christensen et al., 2003; Ronneau and Helaine, 2019), but we previously found in Escherichia coli MG1655 that although stress can drive transcriptional induction, active toxins do not get released (LeRoux et al., 2020). Increasing evidence suggests that some TA systems function in phage defense, which may explain both their variability and ubiquity (Song and Wood, 2020). One of the best characterized examples are type III ToxIN systems, first identified in Pectobacterium atrosepticum, which feature an endoribonuclease toxin, 
ToxN (Fineran et al., 2009). For a ToxIN system found in some E. coli strains, it was recently shown that toxin is liberated following phage-induced shutoff of host transcription and subsequent degradation of the unstable antitoxin (Guegler and Laub, 2021). The toxin then cleaves phage mRNAs to prevent translation of key structural components. Another well-characterized phage defense TA system is RnlAB, a type II system whose toxin is also an RNase (Koga et al., 2011). For most other TA systems that function in phage defense, the toxin's mechanism of action has only been studied by overexpression, not during infection, so how they disrupt the phage life cycle is unclear. However, given the remarkable diversity of biochemical functions ascribed to toxins beyond RNases, TA systems may block phage development at different stages in many different ways.

We set out to identify additional TA systems that provide phage defense by identifying systems frequently found near other phage defense elements. Phage defense systems are often co-located on bacterial chromosomes in so-called defense islands (Makarova et al., 2011, 2013). Efforts to identify genes of unknown function that are frequently found in such genomic contexts has proven to be a fruitful strategy for identifying new phage defense systems (Doron et al., 2018; Gao et al., 2020). Applying this same approach specifically to TA systems led to the identification of two systems, DarTG1 and DarTG2, that can provide E. coli with potent defense against select phage. Previous work on DarTG systems demonstrated that DarT toxins can use NAD+ to ADP ribosylate DNA, and artificial overexpression of these toxins can disrupt chromosomal DNA replication (Jankevicius et al., 2016; Lawarée et al., 2020; Schuller et al., 2021; Zaveri et al., 2020). However, our work now demonstrates that under the natural activating conditions of phage infection, the DarT toxins in fact ADP-ribosylate phage DNA, which inhibits both viral DNA and RNA synthesis. Without new copies of their genomes to package, phage are unable to form progeny. Phage can evolve to overcome DarT activity by two different strategies, either mutating their DNA polymerase, likely to bypass ADPribosylation in the DNA, or by modifying an existing anti-DarT factor. In sum, our work demonstrates that DarTG systems can provide cells with strong defense against phage infection through the ADP-ribosylation of phage DNA. More generally, our work underscores the notion that the enzymatically diverse toxins of TA systems may equip bacteria with a diverse arsenal of phage defense mechanisms. 


\section{Results}

\section{DarTG systems provide defense against phage}

We set out to examine the propensity of ten common type II TA systems to be present in defense islands, a property previously found as predictive for function in phage resistance (Doron et al., 2018). To this end, we analyzed the genomic context of 202,402 toxin genes found in $\sim 38,000$ bacterial and archaeal genomes. For each type of toxin we calculated a 'defense score', calculated as the fraction of toxin homologs found within ten genes of known phage defense genes (Fig. 1A). It was previously shown that defense scores $>0.4$ are strongly predictive of anti-phage activity (Doron et al., 2018). In our analysis, one TA family, the DarTG system, stood out with a defense score of 0.48 , meaning that nearly half of the genes in this family are next to known defense genes in microbial genomes.

DarTG systems have not been previously implicated in phage defense. To test if they can indeed provide defense against phages, we cloned two DarTG systems. In each case, we included the toxin and antitoxin open reading frames, as well as the native, upstream region encompassing the promoter. These systems were cloned into a pBR322 vector backbone and transformed into E. coli MG1655. Each system was then tested against a panel of 12 phage that can infect MG1655 in both fast and slow growth conditions (LB medium at $37{ }^{\circ} \mathrm{C}$, fast growth; M9-glucose medium at $30^{\circ} \mathrm{C}$, slow growth) (Fig. 1B-D). Both systems provided robust defense against different phages under different conditions. DarTG1 prevented plaquing of RB69 and T5 in fast growth conditions, while DarTG2 provided robust phage defense against T5, SEC $\phi 18$, and Lust in slow growth conditions, with modest protection against $\mathrm{T} 5$ in fast growth conditions.

The DarTG1 and DarTG2 systems we cloned were not near other known defense systems, but were each within prophages in E. coli strains C7 and 2-460-02_S4_C3, respectively (Fig. 1E). Homologs of the DarT toxins were previously shown to be single-stranded DNA ADP-ribosyltransferases (Jankevicius et al., 2016; Schuller et al., 2021). A multiple sequence alignment of DarT homologs (often annotated as containing DUF4433), including the ones we cloned and those previously characterized biochemically, revealed high similarity across the entire length of the proteins (Fig. 1F, top). There was complete conservation of many residues including a glutamate (E152 and E147 in the cloned DarT1 and DarT2, respectively) (Fig. 1F, red highlight), known to be critical for catalysis of these ssDNA ADP ribosyltransferases (Jankevicius et al., 2016; Schuller et al., 2021). We mutated 
this conserved glutamate to an alanine in both DarT1 and DarT2 and found that phage defense was abolished in each case (Fig. 1C-D). Hereafter, we refer to these inactive, mutant versions of the TA systems as DarT*G1 and DarT*G2.

In contrast to the DarT toxins, a multiple sequence alignment of their cognate DarG antitoxins revealed two distinct families (Fig. 1F, bottom). DarG1 contains a putative YbiA-like fold (80\% confidence, Phyre 2 prediction), a domain predicted to function in ADP-ribose processing (Souza and Aravind, 2012), while DarG2 features a highly conserved, N-terminal macrodomain known to hydrolyze the ADP-ribose modifications introduced by their cognate toxins (Jankevicius et al., 2016; Rack et al., 2016; Tromans-Coia et al., 2021). The C-terminal region of both proteins, which has been implicated in binding directly to DarT2 (Lawarée et al., 2020), is conserved in both protein families.

\section{DarTG-mediated phage defense functions via an abortive infection mechanism}

Phage defense often occurs via an abortive infection (Abi) mechanism in which the infected cell dies but no phage progeny are produced, thereby preventing spread of the virus in a population. One key characteristic of an Abi mechanism is that when most cells are infected at a high multiplicity of infection (MOI), the growth of the bacterial population stops, while at lower MOIs, the uninfected bacteria can continue to grow. To test whether the DarTG systems trigger Abi, we infected cells harboring either the native DarTG1 or the inactive DarT*G1 system with RB69 phage at varying MOIs, and tracked bacterial growth by $\mathrm{OD}_{600}$ over time (Fig. 2A). At MOIs of 1 and 10, cells harboring DarTG1 did not grow and the $\mathrm{OD}_{600}$ of the cultures decreased over time, indicating that cells were lysing. There was a noticeable delay in lysis compared to cells harboring DarT*G1, likely because the production of new, mature virions is coupled to cells lysis (Young et al., 2000), not because a subpopulation of cells survives. Consistent with this interpretation, we found that no viable cells remained 30 min post-infection for either DarTG1 or DarT*G1 containing cells (Fig. 2C). At MOIs of 0.1 and 0.01 , the $\mathrm{OD}_{600}$ of cultures harboring DarTG1, but not DarT*G1, increased over time indicating that cell growth continued as DarTG1 prevents the phage infection from spreading throughout the population. Similar trends were seen for DarTG2 cells infected with T5 (Fig. 2B, 2D). These data suggested that both DarTG1 and DarTG2 provide phage defense via an abortive infection mechanism. 
We also directly assessed the number of RB69 progeny produced in DarTG1 and DarT*G1containing cells. For cells containing the inactive DarT*G1 system, the initial burst occurred about 20 min after infection and released $\sim 50$ phage (Fig. 2E). In contrast, when DarTG1 was present, no phage progeny were detected up to 45 min post-infection. We obtained similar results for DarTG2 infected with T5 (Fig. 2F) although the timing of the burst was slower. Taken all together, our results support an abortive infection mechanism for DarTG-containing cells, in which activation of the toxin effectively thwarts the production of new phage particles, but infected cells do not survive.

We also tracked infected cells by time-lapse fluorescence microscopy, using the cell-permeable DNA dye 4',6-diamidino-2-phenylindole (DAPI) to stain both bacterial and phage DNA. Phage particles appear as extracellular puncta in the DAPI channel and thus phage infections can be visualized in real time, while DAPI staining of host DNA simultaneously allows tracking of bacterial cell lysis. Consistent with the population-level assays above (Fig. 2A-B), we found that lysis was delayed in the DarTG1-containing cells by approximately $10 \mathrm{~min}$ (Fig. 2G). Cellular DNA also appeared compacted in the DarTG1-containing cells prior to lysis (Fig. 2G, 20' time point). In the DarT*G1-containing cells, the DNA appeared more diffuse throughout the infection process, and following lysis, new phage particles appeared (Fig. 2G, red arrowheads). There are some DAPI-stained, extracellular puncta present around both DarTG1 and DarT*G1 cells prior to cell lysis, which are likely unadsorbed phage. The number of these extracellular puncta increased substantially following lysis of cells harboring DarT*G1, but not DarTG1.

We found that T5 infections did not proceed effectively under time-lapse microscopy conditions, so we sampled from liquid cultures of DAPI-stained, T5-infected cells and imaged them at various time points after infection (Fig. 2H). Similar to DarTG1, we saw fewer DarTG2-containing cells lysing combined with a dramatic difference in the appearance of new phage particles following infection of DarT*G2 versus DarTG2-containing cells. These microscopy experiments support an abortive infection mechanism for both DarTG systems and, at least for DarTG1, suggest that DarT may affect DNA.

\section{Activated DarT inhibits DNA synthesis by ADP-ribosylating DNA}

Previous studies demonstrated that ADP-ribosylation of chromosomal DNA by DarT, either following ectopic expression of DarT or the artificial depletion of DarG, inhibits DNA replication in 
E. coli and M. tuberculosis (Jankevicius et al., 2016; Lawarée et al., 2020; Zaveri et al., 2020). However, we hypothesized that after phage infection, its more relevant biological function is to prevent the replication of phage genomes. To test this hypothesis, we first assessed the quantity of DNA extracted from equal numbers of cells containing either DarTG1 or DarT*G1 and infected with RB69. DNA was harvested from cells 15-18 min. post-infection, before lysis occurs (Fig. 2E). Considering only DNA fragments $<200 \mathrm{~kb}$ (which should primarily represent phage DNA), we found that for cells harboring wild-type darTG1 there was $\sim 4$-fold less DNA compared to cells with $\operatorname{darT}^{*} G 1$ (Fig. 3A). Similarly, we found that after T5 infection, about $\sim 3.5$ fold less phage DNA was recovered from cells with DarTG2 compared to those with DarT*G2 (Fig. 3B).

The decrease in overall DNA content following phage infection when DarTG was present prompted us to ask whether DNA synthesis rates were reduced. To this end, we monitored the uptake of radiolabeled thymidine at various time points after RB69 infection. In DarTG1-containing cells, DNA synthesis rates did not substantially increase, particularly compared to cells with DarT*G1 where the levels of thymidine incorporation increased $\sim 15$-fold following phage infection (Fig. 3C). Differences in DNA synthesis rates were detected as early as 5 minutes post-infection, indicating a rapid activation of the DarT1 toxin following phage infection. DarTG2 similarly prevented an increase in DNA synthesis following T5 infection (Fig. 3D). To rule out that these effects of DarTG1 and DarTG2 were simply non-specific, or secondary, effects of an activated phage defense system, we also measured DNA synthesis rates in conditions where the E. coli RnlAB TA system, which contains an RNase toxin, is activated. We infected either wild-type or $\triangle r n l A B$ cells with T4 $\Delta d m d$, a variant of T4 susceptible to defense by the RnlAB system (Otsuka and Yonesaki, 2012). The rate of DNA synthesis was similar in wild-type and $\triangle r n l A B$ cells, supporting the conclusion that DarTG1 and DarTG2 specifically affect phage replication (Fig. 3E).

The thymidine incorporation experiments do not distinguish between synthesis of host and viral DNA. To assess the relative abundance of each, we deep sequenced DNA from cells post-infection. For RB69 infection of cells harboring DarTG1, $\sim 30 \%$ of the total DNA was phage derived, whereas for cells with DarT*G1, phage DNA was $\sim 70 \%$ of the total (Fig. 3F). This result indicates that active DarT1 prevents the accumulation of new phage DNA. We also examined sequencing coverage across the RB69 genome, finding a non-uniform distribution of reads for cells with DarTG1, but not DarT*G1, suggesting that the toxin disrupts DNA replication elongation, not initiation (Fig. 3G). For 
DarTG2 cells infected with T5, DNA sequencing indicated that only $\sim 20 \%$ of reads were phagederived at 20 min post-infection, but $>90 \%$ of reads were viral by $40 \mathrm{~min}$ (Fig. $3 \mathrm{H}$ ). There were no major differences between the DarTG2 and DarT*G2 cells, likely because T5 triggers rapid and complete degradation of the host chromosome in both cases (McCorquodale and Warner, 1988). However, as with DarTG1, there were significant differences in read coverage indicating that DarT2 likely also disrupts phage DNA replication elongation (Fig. 3I).

DarT family toxins have been shown to ADP-ribosylate ssDNA in vitro and when overexpressed in bacterial cells (Jankevicius et al., 2016). We therefore hypothesized that RB69 and T5 infection activates the DarT1 and DarT2 toxins, respectively, and that activated toxin blocks phage replication by ADP-ribosylating DNA in the cell. To assess whether DarTG-dependent ADP-ribosylation of DNA occurs in vivo following phage infection, we adapted a recently developed technique for measuring ADP-ribosylation of proteins to measure ADP ribosylation of DNA. This assay, enzymatic labeling of terminal ADP-ribose (ELTA), leverages the enzymatic activity of the innate immune protein, OAS1, to covalently attach dATP to ADP-ribose moieties (Ando et al., 2019) (Fig. 3J). This assay has not previously been used to assess the ADP-ribosylation of DNA. Therefore, we first confirmed that purified ADP-ribosylated ssDNA produced robust signal in an ELTA assay (Fig. 3K).

We infected cells harboring darTG1 or $\operatorname{darT}^{*} G 1$ with RB69, isolated DNA and then added OAS1 and ${ }^{32} \mathrm{P}-\mathrm{dATP}$ to label ADP-ribose groups on the DNA (Fig. 3J). We detected a 6.5-fold increase in ADP-ribosylation of DNA extracted from DarTG1-containing cells 20 min post-infection compared to cells containing the inactive DarT1* variant (Fig. 3L). We also measured ADP-ribosylation of DNA following T5 infection of cells harboring darTG2, and found a similar, 4.1-fold increase in ADP-ribosylation in DarTG2 vs DarT*G2-containing cells after 20 min of infection (Fig. 3M). We also observed an 5.7-fold increase in ADP-ribosylation of DNA after 40 min of infection when our sequencing indicated that virtually all DNA was of phage origin (Fig. 3H,M). Taken all together, our results indicate that DarT toxins are rapidly activated following phage infection and ADP ribosylate phage DNA to disrupt its replication.

\section{Activated DarT also inhibits RNA synthesis and phage protein production patterns}

We also asked whether the DarTG systems impact RNA and protein synthesis. RNA synthesis rates were assayed by monitoring radiolabeled uridine uptake following infection. RNA synthesis was 
significantly reduced in both DarTG1- and DarTG2- containing cells following infection with RB69 and T5, respectively (Figs. 4A-B). To assess how DarTG1 and DarTG2 affect protein synthesis, we measured the incorporation of radiolabeled cysteine and methionine, using SDS-PAGE to resolve individual proteins over time post-infection. In contrast to DNA and RNA synthesis, protein synthesis rates did not differ substantially between DarTG1 and DarT*G1-containing cells infected with RB69 (Fig. 4C). However, there was a shift in which proteins were being synthesized, starting around 10 minutes post-infection. In particular, late-appearing species in DarT*G1-infected cells were either not seen or were significantly reduced in DarTG1-infected cells, with many early-appearing species persisting throughout the 20 min time course. For DarTG2 and DarT*G2-containing cells infected with T5, the overall protein synthesis rates were again not substantially different (Fig. 4D). However, in this case, the banding pattern of DarTG2 and DarT*G2 cells remained more similar throughout the 40 min time course, though at the latest time points, DarTG2 cells appear to still be producing more middle gene products and less late gene products. Taken together, our results indicate that upon phage infection, both DarT toxins rapidly block DNA synthesis, and reduce RNA synthesis. Protein synthesis rates are not significantly affected, but the timing and identities of proteins synthesized are misregulated. We conclude that DarT activation disrupts the proper development of mature virions. Even if phage capsids are produced, the inhibition of DNA synthesis means no new phage genomes are available for packaging.

\section{A single point mutation in 61.2 allows RB69 phage to escape DarTG1 defense}

To gain additional insight into how DarTG systems function, we asked how phage can evolve to escape or overcome DarTG-mediated defense. To evolve resistant populations of phage, we serially passaged RB69 on both DarTG1 and DarT*G1-containing cells and monitored the pooled phage population for changes in susceptibility to DarTG1 (Fig. 5A). By pooling together phage propagated on DarTG1 and DarT*G1, we were able to increase the diversity of the population, which cannot normally replicate on DarTG1-containing cells. We isolated a DarTG1-resistant clone from each of five different, independently evolved populations (Fig. 5B) and sequenced their genomes. For one clone, we could not identify any mutations, but the other four DarTG1-resistant clones each had a mutation in the same codon of the uncharacterized gene 61.2, which is predicted to encode a 212 amino acid protein. In three cases, the mutation results in a substitution of arginine-164 with a histidine, while in the fourth arginine-164 becomes a serine (Fig. 5B). 
Intriguingly, a multiple sequence alignment of 124 homologs of gp61.2, which are encoded in both phage and bacterial genomes, revealed that histidine and serine, as well as asparagine, occur naturally at this position (Fig. 5C). Indeed, two phage (T4 and T6) closely related to RB69 that we found DarTG1 did not defend against (Fig. 1B) both encode homologs of gp61.2 containing a histidine at this position of gp61.2. Additionally, we noted that 61.2 is close within the RB69 genome to $d m d$, an inhibitor of the RnlA toxin of the RnlAB toxin-antitoxin system (Otsuka and Yonesaki, 2012). Together, these observations suggest that 61.2 homologs encode inhibitors of DarT toxins with the identity of position 164 strongly influencing the specificity of inhibition. To test this hypothesis, we asked whether ectopic expression of gp61.2(R164H) from evolved RB69 or the gp61.2 homolog from T4 could restore the ability of wild-type RB69 to infect cells containing DarTG1. Indeed, expressing either of these constructs improved the EOP of RB69 and led to more significant plaque clearing compared to cells harboring an empty vector or expressing the wild-type gp61.2 from RB69 (Fig. 5D). When induced in this manner, RB69 gp61.2(R164H) and T4 gp61.2 did not fully restore the infectivity of RB69 suggesting that the timing or levels of expression of gp61.2 may be important for function, or that additional phage factors must be present for full activity. We therefore assessed DarT1 toxin activity when cells were infected with evolved RB69 phage by measuring DNA ADPribosylation levels, reasoning that if DarT1 is inhibited by the gp61.2(R164H) variant, the toxin should be unable to ADP-ribosylate DNA. Consistent with this idea, there was no detectable DNA ADP-ribosylation in cells infected with the evolved RB69 producing the gp61.2(R164H) variant (Fig. 5E). Taken together, these results indicate that 61.2 likely encodes a DarT inhibitor and that the escape mutants we isolated improve the ability of the RB69 gp61.2 to inhibit DarT1.

\section{Modification of a hypothetical gene and DNA polymerase enables escape from DarTG2 defense}

We also tried to evolve T5 phage to escape DarTG2-mediated defense, but were unable to identify escape mutants, even after several independent populations were passaged for 15 cycles each. We therefore tried to evolve SEC $\phi 18$ phage, which DarTG2 also defended against (Fig. 1B). In this case, resistant SEC $\phi 18$ populations were readily obtained, with increased infectivity emerging after 5 rounds of passaging (Fig. 5F). Full genome sequencing of the evolved escape phage populations revealed that each population had accumulated one or two mutations in a hypothetical gene, mga32, and one of five different mutations in mga42, which encodes the SEC $\phi 18$ DNA polymerase (Fig. 5F). The mutations in DNA polymerase did not obviously cluster when mapped onto a homology modelled 
structure of the protein. Although the resulting substitutions in DNA polymerase differed among escape populations, mga32 was always mutated to D40G and, in four cases, also had the additional mutation Q86R. Mga32 has no predicted function or structure.

There are three possibilities for how these mutations allow SEC $\phi 18$ phage to escape the DarTG2 system: (1) one or both of the wild-type phage proteins normally activates the toxin, with the mutant variant having lost the ability to do so; (2) the mutant, but not wild-type, variant of the protein neutralizes the toxin; or (3) the mutant protein overcomes or somehow circumvents the activity of the toxin. We ruled out possibility 1 by overexpressing both wild-type mga32 and mga42 in cells containing the DarTG2 system, and found that these genes did not induce toxicity indicating that they are not sufficient to activate DarT2 (Fig. 5G). We addressed possibilities (2) and (3) by asking whether the DarT2 toxin was still able to ADP-ribosylate DNA following infection with the SEC $\phi 18$ escape phage. If ADP-ribosylation of DNA still occurs in the presence of the evolved phage, it would indicate that the DarT2 toxin is still active but that phage can replicate despite its activity. Indeed, we found that ADP-ribosylation of DNA occurs at a rate similar to that seen with wild-type SEC $\phi 18$ (Fig. 5H). This is in contrast to what we had observed when evolved RB69 infected DarTG1 cells (Fig. 3E), further underscoring that these phage have overcome DarTG-mediated defense by different mechanisms. Whether mutations in both mga32 and mga42 are required for this effect is not yet clear. Nevertheless, these results strongly support a model in which SEC $\phi 18$ modifies its DNA polymerase to accommodate the ADP-ribose modifications on the DNA made by DarT2. 


\section{Discussion}

\section{DarTG-based phage defense}

Like most TA systems, the DarTG system had previously only been characterized through the artificial overexpression of DarT toxin or the depletion of its antitoxin (Jankevicius et al., 2016; Lawarée et al., 2020; Zaveri et al., 2020). This prior work elucidated the biochemical function of DarT toxins as ADP-ribosyltransferases that specifically target DNA and demonstrated that cells lacking DarG mount a DNA damage response as the ADP-ribosylation of DNA by DarT likely leads to replication fork stalling (Tromans-Coia et al., 2021). However, the physiological function and native triggers of DarTG systems has been unclear. A Mycobacterium tuberculosis strain lacking $\operatorname{darTG}$ was reported to have a modest increase in growth after 15 days, though whether the growth defect of the wild-type results from ADP-ribosylation of DNA was not shown and why cells harbor a system that slows growth is not obvious. Here, we demonstrated that DarTG1 and DarTG2, taken from two strains of $E$. coli and representing the two different, major classes of DarTG systems, can provide host cells with potent defense against phages (Fig. 1).

The identification of phages that trigger these DarTG systems enabled us to characterize the activity of the toxins in vivo under growth conditions that naturally, and rapidly, liberate them. Our results indicate that within minutes after phage adsorb to the cell and inject their DNA, DarT toxins are active (Fig. 6). The liberated DarT then ADP-ribosylates phage DNA leading to a near complete cessation of DNA synthesis and an inhibition of RNA synthesis. These effects of the DarT toxins had variable impact on the program of phage gene expression and the production of late proteins (Fig. 4). However, even if procapsids did assemble, DNA packaging could not occur as the phage genome is not fully replicated. Packaging of DNA and capsid completion are required for most phage to initiate lysis of their host cell, likely explaining the delayed lysis of infected DarTG+ cells (Young et al., 2000). Such cells are, however, not viable (Fig. 2C-D), supporting a model in which DarTG functions through an Abi-like mechanism in which infected cells die, but without producing progeny phage, thereby sparing uninfected neighbor cells. Although DarT can, when overexpressed, also target the host cell's chromosome, T5 and likely RB69, trigger chromosome degradation (Petrov et al., 2010; Warner et al., 1975). Thus, we conclude that DarTG does not ultimately kill the host cell, as in a conventional Abi mechanism, and instead acts to thwart phage replication directly. 


\section{Activation of the DarTG system by phage infection}

How DarT becomes activated following phage infection is not yet clear. As noted above, DarT is active within 5 minutes post-infection, as measured by differences in DNA synthesis rates between DarTG and DarT*G cells (Fig. 3C), but without significant transcriptional induction of the system. Recent work on an E. coli ToxIN system demonstrated that the antitoxin toxI is intrinsically unstable and so must be continuously produced to bind and neutralize ToxN (Guegler and Laub, 2021). Phageinduced shutdown of host transcription following T4 infection leads to the liberation of ToxN, an endoribonuclease, $\sim 20$ min post-infection. DarT activation could work similarly. However, although the antitoxins of TA systems are often more unstable than their cognate toxins, we recently found that many are not unstable enough to produce pools of active toxin on the time-scale of a phage infection simply by blocking transcription (LeRoux et al., 2020). Also, we did not find either DarTG system to protect against $\mathrm{T} 4$, which rapidly and almost completely shuts down host transcription following infection. Thus, we favor the possibility that a particular phage protein or factor somehow triggers activation of DarT. The involvement of a specific phage protein would potentially explain why DarTG1 and DarTG2 protect against different phage (Fig. 1B) and it might also explain why different growth conditions are required for their activity. Activation may require particular growth conditions if, for example, a host factor is required for releasing the toxin, but that factor is less abundant or less available in particular growth states.

If a specific phage protein is required to trigger DarT, it could stimulate separation of DarT from its cognate DarG antitoxin. However, the DarG antitoxins may not only bind and inhibit their cognate DarT toxins, as with canonical type II TA systems. Prior work on the enteropathogenic E. coli DarTG system indicated that these systems may also rely on a type IV mechanism in which the antitoxin indirectly antagonizes the toxin's activity (Lawarée et al., 2020). Notably, DarG1 contains structural homology to a YbiA-like domain that is predicted to be an ADP-ribose processing enzyme and DarG2 contains a classic macrodomain typically associated with ADP-glycohydrolases known to remove ADP-ribose modifications (Rack et al., 2016; Souza and Aravind, 2012). Thus, in principle, DarT may always be active, but with sufficient DarG activity in uninfected cells to offset its toxicity. A phage product could inhibit, sequester, or degrade DarG, enabling the rapid accumulation of DarT activity. 


\section{Phage evasion of DarTG-mediated defense}

Phage and their hosts are locked in an arms race wherein hosts acquire or evolve protection from phage infection, and phage evolve mechanisms to overcome these defenses. By evolving RB69 to escape the defense offered by DarTG1, we identified 61.2 as a likely phage-encoded anti-DarT factor. Single substitutions in gp61.2 enabled RB69 phage to overcome DarTG1. Notably, the mutations selected for in gp61.2 convert arginine 164 to histidine or serine, which occur naturally at the equivalent position in the gp61.2 homologs of T4 and T6. DarTG1 did not protect against these Teven phages, possibly because they encode gp61.2 homologs that can already overcome this particular DarTG1 system. The presence of 61.2 homologs in T4, T6, RB69, and other T-even phages suggests that these phages have all been exposed to and selected to produce anti-DarT proteins, underscoring the notion that DarTG systems are critical and likely common phage defense systems in E. coli and possibly many other species. The 61.2 homologs are all found in similar genomic positions in the genomes of T-even phages and relatively close to $d m d$ homologs that encode for RnlA toxin inhibitors. Thus, this region may represent an anti-TA system island, similar to the anti-CRISPR islands documented in mobile genetic elements of Enterobacteriaceae and Pseudomonas (PinillaRedondo et al., 2020).

For SEC $\phi 18$, the mutants that escape defense by DarTG2 all harbored point mutations in two genes, $m g a 32$ and $m g a 42$ (Fig. 5G). The mutations in $m g a 42$, which encodes the phage DNA polymerase, are likely to be important for escape given that DarT2 disrupts DNA replication. Our ELTA measurements indicated that the DNA of escape phage is ADP ribosylated to a similar extent as wildtype phage (Fig. 5H) suggesting that the mutations in mga32 and mga42 are not leading to a disruption of DarT2 activity. Instead, they somehow promote the replication of modified DNA, but how this occurs remains to be studied. Additionally, whether the mutations in mga32 and mga42 are both required is not clear as we have not yet isolated phage that contain only one of the two mutations.

\section{TA systems in phage defense}

Our bioinformatic screen indicated that DarTG homologs are often found in phage defense islands. This result further supports the notion that proximity to known phage defense elements is a powerful means of identifying new phage defense systems (Doron et al., 2018; Makarova et al., 2011). Intriguingly, the RnlAB family, which is known to consist of phage defense systems RnlAB and 
LsoAB, had the lowest phage defense score measured (Otsuka and Yonesaki, 2012) (Fig. 1A). This could indicate that only a subset of the RnlAB family are involved in phage defense. The two DarTG systems we examined are not encoded near any known or predicted phage defense system and instead are encoded within prophages (Fig. 1E). Prior work has found that prophages often harbor phage defense systems (Bondy-Denomy et al., 2019; Dedrick et al., 2017; Rousset et al., 2021). The presence of TA systems on prophage has also been documented, and in some cases these systems may stabilize prophages within the bacterial chromosome, analogous to the role of some TA systems in stabilizing plasmids (Hallez et al., 2010; Peltier et al., 2020; Yao et al., 2018). However, the primary, or perhaps additional function, of these prophage-associated TA systems may be to exclude other phage, which in threatening the host cell, also jeopardize their survival and propagation.

Identifying additional TA systems that are associated with defense islands or encoded within prophage could help to identify additional systems that function in phage-defense. These systems may provide cells a diverse arsenal of anti-phage elements that complement restriction-modification and CRISPR systems. Importantly, those latter systems can only block phages by degrading phage DNA or RNA, whereas TA systems employ toxins with a wide range of enzymatic activities, including the DarT toxins we found here to ADP-ribosylate phage DNA. 


\section{Acknowledgements}

M. LeRoux is supported by a postdoctoral fellowship from the Charles A. King Trust Postdoctoral Research Fellowship Program, Bank of America, N.A., Co-Trustees. This work was funded by an NIH grant to M.T.L. (R01GM082899), who is also an Investigator of the Howard Hughes Medical Institute. This work was also supported by the Sagol Weizmann-MIT Bridge Program (M.T.L. and R.S.) and grants to R.S.: the European Research Council (grant ERC-CoG 681203), the Ernest and Bonnie Beutler Research Program of Excellence in Genomic Medicine, and the German Research Council (DFG) priority program SPP 2330 (grant SO 1611/2). A.K.L.L. was supported by an NIH grant (R01GM104135). 


\section{Methods}

\section{Bioinformatic analysis of TA systems association with defense islands}

Protein sequences of all genes in a set of 38,167 bacterial and archaeal genomes were downloaded from the Integrated Microbial Genomes (IMG) database (Chen et al., 2019) in October 2017. These proteins were clustered using the 'cluster' option of MMseqs2 (release 2-1c7a89 (Steinegger and Söding, 2017)), with default parameters. Clusters were further aggregated into larger clusters using four additional cycles of clustering, in which — in each cycle — a representative sequence was taken from each cluster using the 'createsubdb' option of MMseqs2 and representative sequences were clustered using the 'cluster' option with the '-add-self-matches' parameter. For the first additional clustering cycle, the 'cluster' option was run with default parameters; for the additional cycles 2-4, clustering was run with sensitivity parameter '-s 7.5', and for the additional cycle 4, the '-clustermode 1' parameter was also added.

Each cluster with 20 or more genes was annotated with the most common pfam, COG, and IMG product annotations in the cluster. For each toxin belonging to one of the analyzed toxin families, genes from all clusters annotated as containing this toxin were aggregated. For each of the toxins families, the fraction of genes that have known defense genes in their genomic environment spanning 10 genes upstream and downstream the inspected gene was recorded. Defense score for each toxin family was calculated as previously described, except that genes belonging to TA systems were eliminated from the positive set (Doron et al., 2018).

\section{Analysis of DarTG1 and DarTG2 genome context and multiple sequence alignments}

A blastp search was seeded with either DarT1 or DarT2. Resulting hits with e-values $<10^{-50}$ were selected and associated nucleotide sequences were obtained. Prophage regions were identified as those with $>50 \%$ of genes having annotated phage elements (e.g. genes predicted to encode integrases, recombinases, phage tail or phage capsid proteins) or by using the Phaster phage identification web tool (www.phaster.ca). Phage defense elements were identified by referencing Genbank annotations with known phage defense systems (e.g. restriction-modification, abortive infection).

Representative examples of DarTG1 systems were identified through a blastp search seeded with DarG1 with e-values $<10^{-50}$. Because of conserved gene order ( $\operatorname{dar} T$ always preceding $\operatorname{dar} G$ ), the 
protein sequence of the protein upstream was used for the DarT alignments. For DarTG2, systems were identified from previous studies (Jankevicius et al., 2016; Lawarée et al., 2020; Zaveri et al., 2020), or through a similar strategy described for DarTG1. Protein sequences were aligned in Geneious v. 2020.0 (Biomatters) using the Clustal Omega algorithm and the resulting image files were exported. Annotations for DarG protein domains were identified through a BLAST (macrodomain) or Phyre 2 structural prediction (YbiA-like, 80\% confidence).

\section{Strains and growth conditions}

All bacterial and phage strains are listed in Table S1. Escherichia coli was grown at $37{ }^{\circ} \mathrm{C}$ in LB medium for routine maintenance and cloning. Phage were propagated by infecting E. coli MG1655 or E. coli MG1655 $\Delta r n l A B$ (T4 $\Delta d m d$ only) cultures of OD 0.1-0.3 at an MOI of 0.1 and incubated with aeration at $37^{\circ} \mathrm{C}$. Following clearing, any remaining cells were pelleted by centrifugation and lysates were filtered through a $0.22 \mu \mathrm{M}$ filter. SEC $\phi 18$ was concentrated for ELTA experiments by centrifugation of cleared lysates for $2 \mathrm{hrs}$ at $10,000 \mathrm{x} \mathrm{g}$ and resulting pellets were resuspended in $\sim 100 x$ less volume.

All RB69 infection experiments were performed $37^{\circ} \mathrm{C}$ in LB medium, whereas experiments with T5 and SEC $\phi 18$ were performed at 30 and $20{ }^{\circ} \mathrm{C}$, respectively, in $\mathrm{M} 9$ medium $\left(6.4 \mathrm{~g} / \mathrm{L} \mathrm{Na}_{2} \mathrm{HPO}_{4}-7 \mathrm{H}_{2} \mathrm{O}\right.$, $1.5 \mathrm{~g} / \mathrm{L} \mathrm{KH}_{2} \mathrm{PO}_{4}, 0.25 \mathrm{~g} / \mathrm{L} \mathrm{NaCl}, 0.5 \mathrm{~g} / \mathrm{L} \mathrm{NH}_{4} \mathrm{Cl}$ medium supplemented with $0.1 \%$ casamino acids, $0.4 \%$ glucose, $2 \mathrm{mM} \mathrm{MgSO}$, and $0.1 \mathrm{mM} \mathrm{CaCl}_{2}$ ), and overnight cultures for experiments were prepared in the same media used for the experiment. Media for selection or plasmid maintenance were supplemented with carbenicillin $(100 \mu \mathrm{g} / \mathrm{mL})$, chloramphenicol $(20 \mu \mathrm{g} / \mathrm{mL})$, or kanamycin $(30$ $\mu \mathrm{g} / \mathrm{ml})$ as necessary unless otherwise indicated. Induction of ectopic expression were effected with anhydrous tetracycline $(100 \mathrm{ng} / \mu \mathrm{L})$, arabinose $(0.2 \% \mathrm{w} / \mathrm{v})$, or vanillate $(100 \mu \mathrm{M})$, as necessary.

\section{Plasmid construction}

All primer and synthesized gene sequences are listed in Table S2. DNA encoding candidate DarTG1 and DarTG2 system open reading frames as well as $200 \mathrm{bp}$ upstream and overlapping ends to the pBR322 vector (MLR1 and MLR2, respectively) were commercially synthesized by Integrated DNA Technology as gBlocks and assembled into a promoter-less backbone of pBR322 amplified with

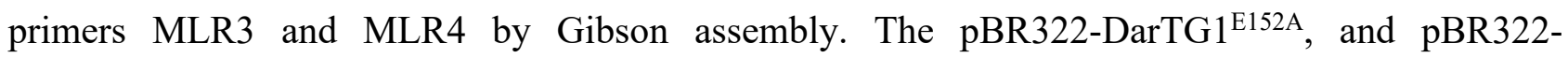
DarTG2 $\mathrm{E}^{147 \mathrm{~A}}$ variants were inserted into the corresponding wild-type plasmids by site-directed 
mutagenesis with inverse PCR using primers MLR5 and MLR6, or MLR7 and MLR8, respectively. The mga32 open reading frame was amplified from either the wild-type SEC $\phi 18$ or an evolved phage clone from population 2 with primers MLR9 and MLR10 and cloned into a version of pKVS45 containing a chloramphenicol resistance cassette, vanillate promoter, and SC101 origin of replication, linearized by PCR amplification with primers MLR11 and MLR12. The mga42 open reading frame was similarly amplified from wild-type or evolved SEC $\phi 18$ with primers MLR13 and MLR14 and cloned via Gibson assembly into a version of pBAD33 with a kanamycin resistance cassette linearized with MLR15 and MLR16. The gp61.2 open reading frame was amplified from either the wild-type RB69 or an evolved clone from population 1 with primers MLR17 and MLR18, or from T4 phage with primers MLR19 and MLR20 and cloned into pKSV45-P van-kan linearized with primers MLR12 and MLR21.

\section{Strain construction}

Plasmids described above were introduced into MG1655 by TSS transformation or electroporation (Chung et al., 1989). A scarless, unmarked deletion of $r n l A B$ was constructed by two-step allelic exchange. The $s a c B-n e o R$ cassette from pIB279 amplified with ML22 and ML23 and inserted using lambda Red recombinase (Blomfield et al., 1991; Datsenko and Wanner, 2000). The deletion oligo (MLR24) was subsequently transformed into lambda Red-containing cells and counterselection was performed on $5 \%$ sucrose plates as previously described. Mutations were verified by PCR amplification and sequencing of sucrose-sensitive, kanamycin-sensitive clones.

\section{Plaque assays, phage titering, and efficiency of plating (EOP) measurements}

Overnight cultures of the indicated bacterial cells were mixed 1:200 with melted 0.5\% agar made with either LB or M9 medium. The agar was then overlaid onto a 1.2\% agar plate composed of the corresponding medium. Ten-fold serial dilutions of the indicated phage were spotted on top. For RB69 experiments, plates composed of LB medium were incubated at $37^{\circ} \mathrm{C}$, whereas for T5 and SEC $\phi 18$ experiments, plates were composed of M9 medium and incubated at 30 and $20{ }^{\circ} \mathrm{C}$, respectively. All experiments were performed independently at least 3 times.

\section{Growth curves}

E. coli bearing the indicated DarTG plasmids were grown to an $\mathrm{OD}_{600}$ of 0.3 and then mixed with phage at the indicated MOIs. A $100 \mu \mathrm{L}$ aliquot of the mixtures were seeded into 96-well plates, and 
growth was measured at 15 min intervals with orbital shaking on a plate reader (Biotek) at $37^{\circ} \mathrm{C}$ for DarTG1-RB69 experiments and $30^{\circ} \mathrm{C}$ for DarTG2-T5 experiments. For growth following DarT1 and expression of gp61.2 variants, overnight cultures of the indicated strains were diluted 1:100 in LB media containing appropriate antibiotics, vanillate $(100 \mu \mathrm{M})$, and arabinose $(0.2 \%)$. Plates were seeded as described for phage infections and data were collected at $37{ }^{\circ} \mathrm{C}$. Representative data presented are the result of 6-12 plate replicates and were replicated independently at least 2 times.

\section{Bacterial survival after phage infection}

E. coli MG1655 containing either DarTG1 plasmids (pMLR4, pMLR5) or DarTG2 plasmids (pMLR6, pMLR7) were grown to $\mathrm{OD}_{600}$ of 0.3 and then phage was added at an MOI of 5 for the RB69 experiments and MOI of 20 for the T5 experiments. Suspensions were incubated without shaking for $5 \mathrm{~min}$ to allow for adsorption, then cultures were washed twice with an equal volume of media to remove unadsorbed phage. Infected cells were incubated at the appropriate temperatures for an additional 5-10 min before serial dilutions were plated on LB plates to determine bacterial colony forming units. Data presented are the mean and standard deviation of 3 biological replicates.

\section{One-step growth curves}

Bacterial cells with appropriate plasmids were infected at an MOI of 0.01 in LB medium at $37{ }^{\circ} \mathrm{C}$ (darTG1-RB69 experiment and $r n l A B-\mathrm{T} 4 \Delta d m d$ experiment) or in M9 medium at $30{ }^{\circ} \mathrm{C}$ (darTG2-T5 experiment). Samples were collected at regular time intervals, serially diluted, and immediately spotted on $0.5 \%$ LB top agar mixed with a wild-type MG1655 indicator strain to determine plaque forming units/mL. Data presented are the mean and standard deviation of 3 biological replicates.

\section{Microscopy}

Cells were grown under conditions described above to an $\mathrm{OD}_{600}$ of 0.3 , then stained with DAPI (1 $\mu \mathrm{g} / \mathrm{mL}$ ) for $10 \mathrm{~min}$. Cells were then infected with phage at MOI of 5 (RB69) or 20 (T5). For darTG1RB69 experiments, the resulting suspension was washed once to remove unadsorbed phage and $1 \mu \mathrm{L}$ was spotted onto a $1.5 \%$ agarose pad prepared with LB containing DAPI $(1 \mu \mathrm{g} / \mathrm{mL})$ and placed in a $35 \mathrm{~mm}$ glass-bottom dish with $20 \mathrm{~mm}$ micro-well \#0 coverglass (Cellvis) sealed with parafilm. Phasecontrast and epifluorescence images were taken at $10 \mathrm{~min}$ intervals on a Zeiss Observer Z1 microscope using a 100x/1.4 oil immersion objective and an LED-based Colibri illumination system using MetaMorph software (Universal Imaging, PA). 
For darTG2 experiments, the DAPI-stained, infected cells were incubated with shaking at $30{ }^{\circ} \mathrm{C}$ and $1 \mathrm{~mL}$ aliquots were removed at the indicated time points. Cells were washed once to remove unadsorbed phage, spotted on 1.5\% agarose pads prepared with M9 medium, placed on glass slides, and 3-5 fields were immediately imaged. Data presented are representative of at least 2 independent biological replicates.

\section{DNA extractions after phage infection}

A volume of 10-25 mL of cells grown and infected as described above for bacterial survival assays were incubated with aeration until the indicated time points. An equal volume of cells maintained at the same optical density for each experiment were then harvested by vacuum filtration onto a $0.2 \mu \mathrm{M}$ filter and washed once with an equal volume of media to remove unadsorbed phage. Filters were placed in conical tubes and cells were removed by scraping into $1 \mathrm{~mL}$ of media. The resulting bacterial suspension was pelleted in a microcentrifuge and the cells were flash-frozen in liquid nitrogen and stored at $-80{ }^{\circ} \mathrm{C}$. DNA was extracted using the Gentra Puregene Kit (Qiagen) and an equal volume of DNA was loaded for each sample and visualized on a $0.5 \%$ agarose gel stained with ethidium bromide. The amount of DNA was quantified in ImageJ (http://imagej.nih.gov/). A representative image is shown of at least two independent biological replicates.

\section{Incorporation assays}

Bacterial cells were infected under the same conditions as described for bacterial survival assays and flasks were maintained with aeration at the appropriate temperature. For DNA and RNA synthesis measurements, at the indicated time points an aliquot of the bacterial culture was transferred to a microcentrifuge tube containing methyl- ${ }^{3} \mathrm{H}$ thymidine (Perkin-Elmer) $(40 \mu \mathrm{Ci} / \mathrm{mL})$ or uridine, 5$6\left[{ }^{3} \mathrm{H}\right]$ (Perkin Elmer) $(6 \mu \mathrm{Ci} / \mathrm{mL})$. Tubes were incubated at the same temperature as infected cultures for $1 \mathrm{~min}\left(37^{\circ} \mathrm{C}\right.$ growth) or $2 \mathrm{~min}\left(30^{\circ} \mathrm{C}\right.$ growth). Reactions were quenched by addition of nonradioactive thymidine or uridine $(1.5 \mathrm{mM})$ and incubated an additional 1 or $2 \mathrm{~min}$. Samples were added to ice cold trichloroacetic acid (TCA) (10\% w/v) and incubated at least $30 \mathrm{~min}$ on ice to allow for precipitation. The resulting sample was vacuum filtered onto a glass microfiber filter (Whatman, 1820-024) that had been pre-wetted with 5\% w/v TCA. Filters were washed with $35 \mathrm{x}$ volume of 5\% w/v TCA, then with $5 \mathrm{x}$ volume of $100 \%$ ethanol. Air dried filters were placed in tubes with scintillation fluid and measured in a scintillation counter (Perkin Elmer). Three independent biological replicates are presented. 
For protein synthesis assays, a $1 \mathrm{~mL}$ aliquot of infected bacterial cultures was removed and incubated with EasyTagTM EXPRESS ${ }^{35} \mathrm{~S}$ Protein Labeling Mix, $\left[{ }^{35} \mathrm{~S}\right]$ (Perkin Elmer) at $44 \mu \mathrm{Ci} / \mathrm{mL}$ for 2 min $\left(37^{\circ} \mathrm{C}\right)$ or $10 \mathrm{~min}\left(30^{\circ} \mathrm{C}\right)$. Labeling was quenched with addition of unlabeled cysteine and methionine at $3 \mathrm{mM}$, and proteins were precipitated by addition of ice cold TCA $(13 \% \mathrm{w} / \mathrm{v})$ and incubation on ice for at least $30 \mathrm{~min}$. Samples were pelleted, washed twice with $100 \%$ acetone, then resuspended in resuspension buffer (100 mM Tris (pH 11.0), 3\% w/v SDS). Samples were resolved by 4-20\% SDSPAGE, after which the gel was soaked in Gel Dry Drying Solution (Thermo Fisher), dried on a vacuum dry gel dryer, and exposed to a phosphorimaging screen for 1-4 days. The screen was imaged on a Typhoon scanner (GE Healthcare) at $50 \mu \mathrm{M}$ resolution. A representative image of two independent experiments is presented.

\section{DNA sequencing}

DNA was collected as described above, then sheared in a Diagenode Bioruptor 300 sonicator water bath for $15 \times 10 \mathrm{~s}$ cycles at maximum intensity. The resulting samples were further purified with a Zymo PCR cleanup kit and concentrations were determined on a Nanodrop. Sequencing libraries were prepared as described previously (Culviner and Laub, 2018) and run on either an Illumina MiSeq or NextSeq500 at MIT BioMicroCenter.

Reads were trimmed and mapped using Geneious 2020.0 (Biomatters) with the Geneious mediumsensitivity mapping to both MG1655 and phage reference genomes. The percent of reads mapping to the phage genome was calculated as (reads mapped to phage/total reads mapped*100) for each sample, and is the average of 2-3 independent biological replicates. Coverage maps were generated by dividing the number of non end-gap characters at each position by the sum of all positions and multiplied by $10^{9}$, then generating a moving average with an interval of 100 . The resulting data were plotted as a function of position. Coverage data presented are from one replicate representative of 23 independent biological replicates.

\section{ELTA assays}

A positive control was generated in vitro via enzymatic ADP-ribosylation of ssDNA as described previously (Jankevicius et al., 2016). Briefly, a $10 \mu \mathrm{L}$ reaction containing $30 \mathrm{ng} / \mu \mathrm{L}$ of DNA substrate containing a TCTC motif (MLR25), $50 \mathrm{mM}$ Tris-HCl pH 8.0, $150 \mathrm{mM} \mathrm{NaCl,} 100 \mu \mathrm{M} \mathrm{NAD}^{+}$, and 30 ng/ $\mu \mathrm{L}$ recombinant Taq DarT (from Thermus aquaticus) was incubated at $37^{\circ} \mathrm{C}$ for $2 \mathrm{hr}$. The reaction 
was purified using the Monarch PCR and DNA cleanup kit (NEB) to remove unincorporated dATP. The successful incorporation of ADP-ribose was confirmed by visualization on 3\% agarose gel after ELTA labeling with Cy5-dATP. The experiment in Fig. 3K was performed with 10 femtomole of the in vitro produced ADP-ribosylated DNA, while the control reaction with non-ribosylated DNA was performed using $1 \mu \mathrm{g}$ of sheared DNA extracted from $E$. coli cells grown to mid-log.

DNA for all other ELTA assays was harvested as described for DNA Extraction after phage infection from cells infected as described for bacterial survival after phage infection experiments. For SEC $\phi 18$ experiments in Figure 5H, cells were infected at an MOI of 1000 due to the low adsorption of this phage under the growth conditions $(<1 \%)$ and infected cultures were incubated for 2 hours prior to harvesting. For all experiments, resulting DNA was sheared for 15 x $30 \mathrm{sec}$ cycles on the BioRuptor Sonicator on the high setting and shearing was confirmed by agarose gel electrophoresis. The resulting sheared samples were further purified with a Zymo DNA Clean and Concentrator kit (Zymo Research).

Each $20 \mu \mathrm{L}$ ELTA reaction consisted of $10 \mathrm{mM}$ Tris- $\mathrm{HCl} \mathrm{pH}$ 7.5, $20 \mathrm{mM}$ magnesium acetate, 2.5 $\mathrm{mM}$ DTT, $10 \mu \mathrm{Ci}(0.05 \mu \mathrm{Ci} / \mu \mathrm{L}) \mathrm{dATP},\left[\alpha^{-32} \mathrm{P}\right]$ (Perkin Elmer), $2 \mu \mathrm{M}$ OAS1, $100 \mu \mathrm{g} / \mathrm{mL}$ low molecular weight poly I:C (Invivogen), and $1 \mu \mathrm{g}$ of DNA. These reactions were incubated at $37^{\circ} \mathrm{C}$ for 2 hours after which the DNA was purified as for the control DNA. Half of the resulting sample was transferred to a $4 \mathrm{~mL}$ scintillation tube and counted as above. Data were normalized to a reaction run with no DNA input. This ADP-ribosylated ssDNA control was run alongside each set of reactions to confirm normal assay function. Two or three independent biological replicates are presented in each graph.

\section{Phage evolution experiments}

Each RB69 evolution experiment consisted of 8 wells of cells producing DarTG1 (ML3) and 8 wells of cells producing DarT* ${ }^{*} 2$ (ML4) seeded with $\sim 10^{6}$ cells/well from overnight bacterial cultures in a 96-well plate in LB medium. Cells were infected with 10-fold serial dilutions of phage cultures with the highest MOI at 10, and one well of each strain uninfected to control for cross-contamination. Plates sealed with a Breathe Easy plate seals (Sigma) and aerated by micro-orbital shaking in an ELTI plate shaker at $37^{\circ} \mathrm{C}$. Plates were incubated for 4-6 hours. The cleared and partially cleared wells were pooled, centrifuged to remove unlysed bacteria, and the resulting supernatants were used to infect the next evolution round. Resistance to DarTG1 was monitored by number of cleared wells as 
well as by EOP assays. Once resistance to DarTG1 was observed, single plaques were isolated in top agar plates and tested for resistance. One resistant clone from each of 5 independently evolved phage populations was propagated for further analysis and sequencing as described below.

The SEC $\phi 18$ evolution experiments were designed as above, except wells were seeded with DarTG2 (ML5) and DarT*G2 (ML6), propagated in M9-glucose medium, and plates were incubated at $20^{\circ} \mathrm{C}$ for $16 \mathrm{hrs}$. Sequencing was performed on evolved populations as opposed to single clones described for the RB69 evolution.

\section{Phage DNA extractions}

Lysed bacterial cultures were centrifuged to remove bacterial cells, then $100 \mu \mathrm{L}$ of supernatant containing phage was incubated with TURBO DNase I $(0.1 \mathrm{mg} / \mathrm{mL})$ and RNase A $(0.1 \mathrm{mg} / \mathrm{mL})$ for $45 \mathrm{~min}$ at $37^{\circ} \mathrm{C}$ to remove residual nucleic acids from bacterial cell lysis. Nucleases were inactivated by addition of EDTA $(10 \mathrm{mM})$ and incubation for $15 \mathrm{~min}$ at $37^{\circ} \mathrm{C}$. Proteinase $\mathrm{K}(0.2 \mathrm{mg} / \mathrm{mL})$ was added and samples incubated at $50{ }^{\circ} \mathrm{C}$ for $30 \mathrm{~min}$ to disrupt phage capsids. Samples were centrifuged to $2 \mathrm{~min}$ at $21000 \mathrm{x} g$ and DNA precipitated from supernatant by standard sodium acetate/ethanol precipitation. The resulting DNA was processed for Illumina sequencing as described above. Mutations were identified using Geneious Variant Finder with the threshold set to $>20 \%$. 


\section{References}

Ando, Y., Elkayam, E., McPherson, R.L., Dasovich, M., Cheng, S.-J., Voorneveld, J., Filippov, D.V., Ong, S.-E., Joshua-Tor, L., and Leung, A.K.L. (2019). ELTA: Enzymatic Labeling of Terminal ADPRibose. Molecular Cell 73, 845-856.e5.

Bernheim, A., Millman, A., Ofir, G., Meitav, G., Avraham, C., Shomar, H., Rosenberg, M.M., Tal, N., Melamed, S., Amitai, G., et al. (2020). Prokaryotic viperins produce diverse antiviral molecules. Nature $\backslash$ ldots 1-25.

Blomfield, I.C., Vaughn, V., Rest, R.F., and Eisenstein, B.I. (1991). Allelic exchange in Escherichia coli using the Bacillus subtilis sacB gene and a temperature-sensitive pSC101 replicon. Molecular Microbiology 5, 1447-1457.

Bondy-Denomy, J., Qian, J., Westra, E.R., Buckling, A., Guttman, D.S., Davidson, A.R., and Maxwell, K.L. (2019). Prophages mediate defense against phage infection through diverse mechanisms. The ISME Journal 10, 1-13.

Chen, I.-M.A., Chu, K., Palaniappan, K., Pillay, M., Ratner, A., Huang, J., Huntemann, M., Varghese, N., White, J.R., Seshadri, R., et al. (2019). IMG/M v.5.0: an integrated data management and comparative analysis system for microbial genomes and microbiomes. Nucleic Acids Research 47, D666-D677.

Christensen, S.K., Pedersen, K., Hansen, F.G., and Gerdes, K. (2003). Toxin-antitoxin Loci as Stressresponse-elements: ChpAK/MazF and ChpBK Cleave Translated RNAs and are Counteracted by tmRNA. Journal of Molecular Biology 332, 809-819.

Chung, C.T., Niemela, S.L., and Miller, R.H. (1989). One-step preparation of competent Escherichia coli: transformation and storage of bacterial cells in the same solution. Proc Natl Acad Sci U S A 86, 2172-2175.

Cohen, D., Melamed, S., Millman, A., Shulman, G., Oppenheimer-Shaanan, Y., Kacen, A., Doron, S., Amitai, G., and Sorek, R. (2019). Cyclic GMP-AMP signalling protects bacteria against viral infection. Nature Vldots 574, 691-695.

Culviner, P.H., and Laub, M.T. (2018). Global Analysis of the E. coli Toxin MazF Reveals Widespread Cleavage of mRNA and the Inhibition of rRNA Maturation and Ribosome Biogenesis. Molecular Cell 70, 868-880.e10.

Datsenko, K.A., and Wanner, B.L. (2000). One-step inactivation of chromosomal genes in Escherichia coli K-12 using PCR products. Proceedings of the National Academy of Sciences 97, $6640-6645$.

Dedrick, R.M., Jacobs-Sera, D., Bustamante, C.A.G., Garlena, R.A., Mavrich, T.N., Pope, W.H., Reyes, J.C.C., Russell, D.A., Adair, T., Alvey, R., et al. (2017). Prophage-mediated defence against viral attack and viral counter-defence. Nature Microbiology 2, 1-13. 
Doron, S., Melamed, S., Ofir, G., Leavitt, A., Lopatina, A., Keren, M., Amitai, G., and Sorek, R. (2018). Systematic discovery of antiphage defense systems in the microbial pangenome. Science (New York, N.Y.) 359, eaar4120-18.

Fineran, P.C., Blower, T.R., Foulds, I.J., Humphreys, D.P., Lilley, K.S., and Salmond, G.P.C. (2009). The phage abortive infection system, ToxIN, functions as a protein-RNA toxin-antitoxin pair. Proc Natl Acad Sci U S A 106, 894-899.

Gao, L., Altae-Tran, H., Böhning, F., Makarova, K.S., Segel, M., Schmid-Burgk, J.L., Koob, J., Wolf, Y.I., Koonin, E.V., and Zhang, F. (2020). Diverse enzymatic activities mediate antiviral immunity in prokaryotes. Science (New York, N.Y.) 369, 1077-1084.

Guegler, C.K., and Laub, M.T. (2021). Shutoff of host transcription triggers a toxin-antitoxin system to cleave phage RNA and abort infection. Mol Cell.

Hallez, R., Geeraerts, D., Sterckx, Y., Mine, N., Loris, R., and Melderen, L.V. (2010). New toxins homologous to ParE belonging to three-component toxin-antitoxin systems in Escherichia coli O157:H7. Molecular Microbiology 76, 719-732.

Harms, A., Brodersen, D.E., Mitarai, N., and Gerdes, K. (2018). Toxins, Targets, and Triggers: An Overview of Toxin-Antitoxin Biology. Molecular Cell 70, 768-784.

Jankevicius, G., Ariza, A., Ahel, M., and Ahel, I. (2016). The Toxin-Antitoxin System DarTG Catalyzes Reversible ADP-Ribosylation of DNA. Molecular Cell 64, 1109-1116.

Koga, M., Otsuka, Y., Lemire, S., and Yonesaki, T. (2011). Escherichia coli rnlA and rnlB Compose a Novel Toxin-Antitoxin System. Genetics 187, 123-130.

Lawarée, E., Jankevicius, G., Cooper, C., Ahel, I., Uphoff, S., and Tang, C.M. (2020). DNA ADPRibosylation Stalls Replication and Is Reversed by RecF-Mediated Homologous Recombination and Nucleotide Excision Repair. Cell Reports 30, 1373-1384.e4.

LeRoux, M., Culviner, P.H., Liu, Y.J., Littlehale, M.L., and Laub, M.T. (2020). Stress Can Induce Transcription of Toxin-Antitoxin Systems without Activating Toxin. Molecular Cell 79, 280-292.e8.

Makarova, K.S., Wolf, Y.I., Snir, S., and Koonin, E.V. (2011). Defense islands in bacterial and archaeal genomes and prediction of novel defense systems. J Bacteriol 193, 6039-6056.

Makarova, K.S., Wolf, Y.I., and Koonin, E.V. (2013). Comparative genomics of defense systems in archaea and bacteria. Nucleic Acids Res 41, 4360-4377.

Malki, K., Sible, E., Cooper, A., Garretto, A., Bruder, K., Watkins, S.C., and Putonti, C. Seven Bacteriophages Isolated from the Female Urinary Microbiota. Genome Announcements 4, e0100316.

McCorquodale, D.J., and Warner, H.R. (1988). Bacteriophage T5 and Related Phages. In The Bacteriophages, R. Calendar, ed. (Boston, MA: Springer US), pp. 439-475. 
Otsuka, Y., and Yonesaki, T. (2012). Dmd of bacteriophage T4 functions as an antitoxin against Escherichia coli LsoA and RnlA toxins. Molecular Microbiology 83, 669-681.

Page, R., and Peti, W. (2016). Toxin-antitoxin systems in bacterial growth arrest and persistence. Nat Chem Biol 12, 208-214.

Peltier, J., Hamiot, A., Garneau, J.R., Boudry, P., Maikova, A., Hajnsdorf, E., Fortier, L.-C., Dupuy, B., and Soutourina, O. (2020). Type I toxin-antitoxin systems contribute to the maintenance of mobile genetic elements in Clostridioides difficile. Commun Biol 3, 1-13.

Petrov, V.M., Ratnayaka, S., Nolan, J.M., Miller, E.S., and Karam, J.D. (2010). Genomes of the T4related bacteriophages as windows on microbial genome evolution. Virology Journal 7, 292.

Pinilla-Redondo, R., Shehreen, S., Marino, N.D., Fagerlund, R.D., Brown, C.M., Sørensen, S.J., Fineran, P.C., and Bondy-Denomy, J. (2020). Discovery of multiple anti-CRISPRs highlights antidefense gene clustering in mobile genetic elements. Nat Commun 11, 5652.

Rack, J.G.M., Perina, D., and Ahel, I. (2016). Macrodomains: Structure, Function, Evolution, and Catalytic Activities. Annu. Rev. Biochem. 85, 431-454.

Ronneau, S., and Helaine, S. (2019). Clarifying the Link between Toxin-Antitoxin Modules and Bacterial Persistence. Journal of Molecular Biology \#startpage\#.

Rousset, F., Dowding, J., Bernheim, A., Rocha, E.P.C., and Bikard, D. (2021). Prophage-encoded hotspots of bacterial immune systems.

Schuller, M., Butler, R.E., Ariza, A., Tromans-Coia, C., Jankevicius, G., Claridge, T.D.W., Kendall, S.L., Goh, S., Stewart, G.R., and Ahel, I. (2021). Molecular basis for DarT ADP-ribosylation of a DNA base. Nature 596, 597-602.

Song, S., and Wood, T.K. (2020). A Primary Physiological Role of Toxin/Antitoxin Systems Is Phage Inhibition. Front. Microbiol. 11.

Souza, R.F. de, and Aravind, L. (2012). Identification of novel components of NAD-utilizing metabolic pathways and prediction of their biochemical functions. Mol. BioSyst. 8, 1661-1677.

Steinegger, M., and Söding, J. (2017). MMseqs2 enables sensitive protein sequence searching for the analysis of massive data sets. Nat Biotechnol 35, 1026-1028.

Tromans-Coia, C., Sanchi, A., Moeller, G.K., Timinszky, G., Lopes, M., and Ahel, I. (2021). TARG1 protects against toxic DNA ADP-ribosylation. Nucleic Acids Res gkab771.

Warner, H.R., Drong, R.F., and Berget, S.M. (1975). Early events after infection of Escherichia coli by bacteriophage T5. Induction of a 5'-nucleotidase activity and excretion of free bases. Journal of Virology 15, 273-280.

Yamaguchi, Y., and Inouye, M. (2011). Regulation of growth and death in Escherichia coli by toxinantitoxin systems. Nat Rev Micro 9, 779-790. 
Yao, J., Guo, Y., Wang, P., Zeng, Z., Li, B., Tang, K., Liu, X., and Wang, X. (2018). Type II toxin/antitoxin system ParESO /CopASO stabilizes prophage CP4So in Shewanella oneidensis. Environ Microbiol 20, 1224-1239.

Young, I., Wang, I., and Roof, W.D. (2000). Phages will out: strategies of host cell lysis. Trends Microbiol 8, 120-128.

Zaveri, A., Wang, R., Botella, L., Sharma, R., Zhu, L., Wallach, J.B., Song, N., Jansen, R.S., Rhee, K.Y., Ehrt, S., et al. (2020). Depletion of the DarG antitoxin in Mycobacterium tuberculosis triggers the DNA-damage response and leads to cell death. Molecular Microbiology 114, 641-652. 
A

\begin{tabular}{c|c|c}
$\begin{array}{c}\text { Toxin } \\
\text { family }\end{array}$ & $\begin{array}{c}\text { analyzed } \\
\text { genes }\end{array}$ & $\begin{array}{c}\% \text { next to known } \\
\text { defense systems }\end{array}$ \\
\hline DarT & 846 & 48 \\
AbiE & 29,617 & 27 \\
HipA & 27,436 & 20 \\
HicA & 39,079 & 17 \\
VapB & 14,143 & 16 \\
Doc & 21,212 & 16 \\
MazF & 38,878 & 15 \\
RelE & 19,500 & 14 \\
HigB & 10,920 & 14 \\
RnIA & 624 & 13
\end{tabular}

B

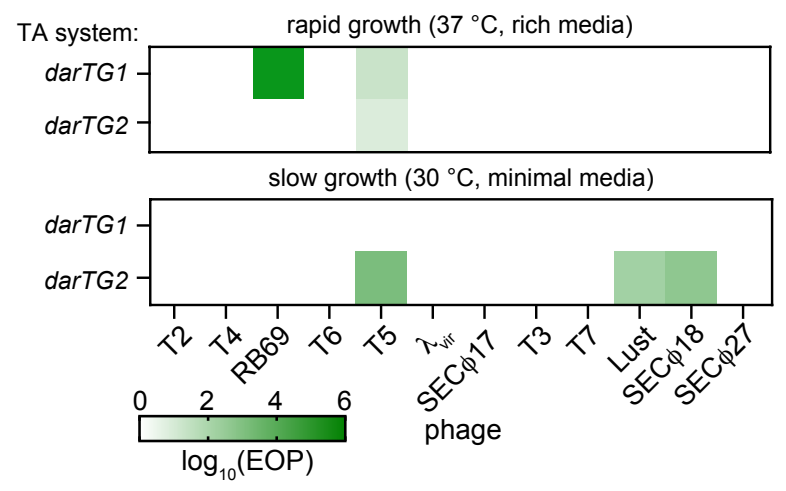

C

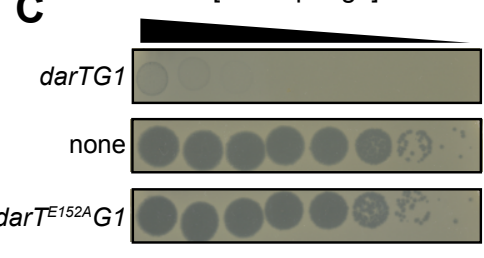

D

[T5 phage]

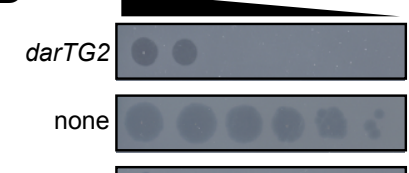

E

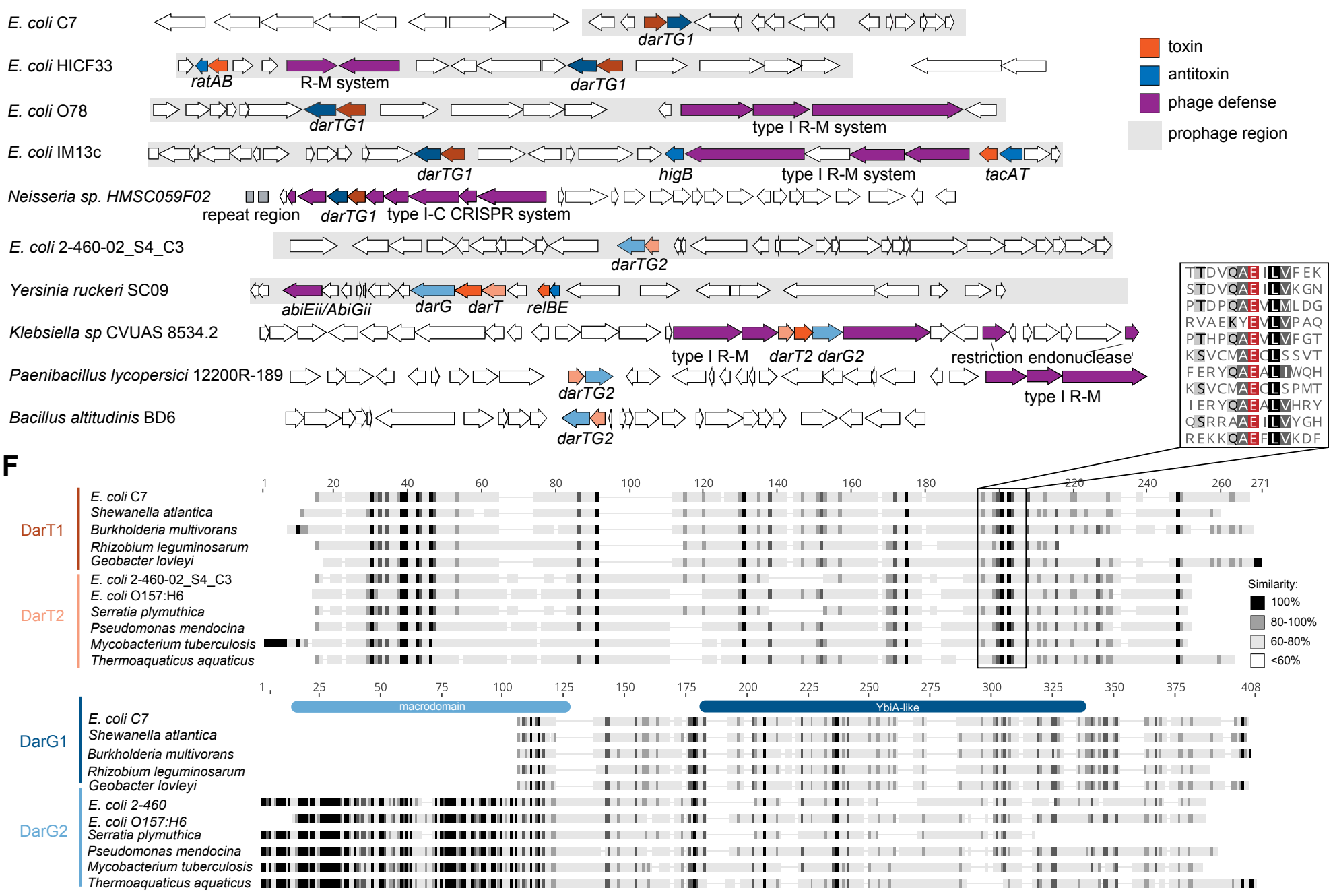

\section{Figure 1. DarTG TA systems defend against phage.}

(A) Defense scores calculated for different TA families.

(B) Efficiency of plaquing (EOP) (indicated in green) for strains bearing the indicated DarTG systems infected with a panel of phage compared to a strain bearing an empty vector under two different growth conditions.

(C-D) 10-fold serial dilution plaque assays of RB69 (C) or T5 (D) phage spotted on E. coli MG1655 harboring the indicated darTG system regulated by its native promoter (top), an empty vector (middle), or the darTG-bearing plasmid with a mutation in the predicted active site of the toxin (bottom).

(E) Genomic context for the darTG1 and darTG2 systems in (B-C), along with additional, selected darTG1-like and darTG2-like systems illustrating their frequent association with prophages and known phage defense elements.

(F) Multiple sequence alignments of representative DarT toxins and DarG antitoxins. 
bioRxiv preprint doi: https://doi.org/10.1101/2021.09.27.462013; this version posted September 27, 2021. The copyright holder for this preprint (which was not certified by peer review) is the author/funder, who has granted bioRxiv a license to display the preprint in perpetuity. It is made.

A

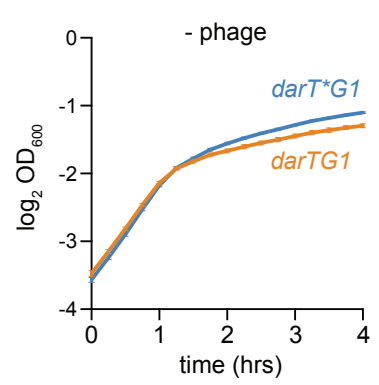

B

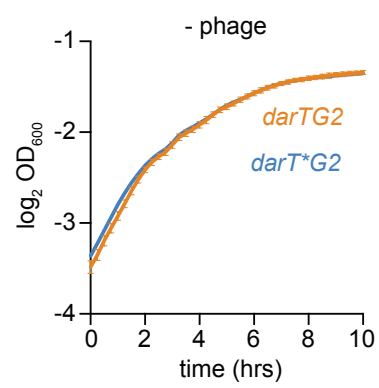

C

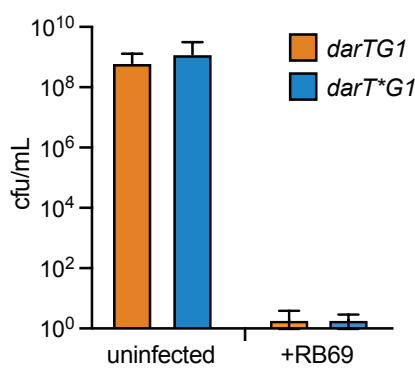

G
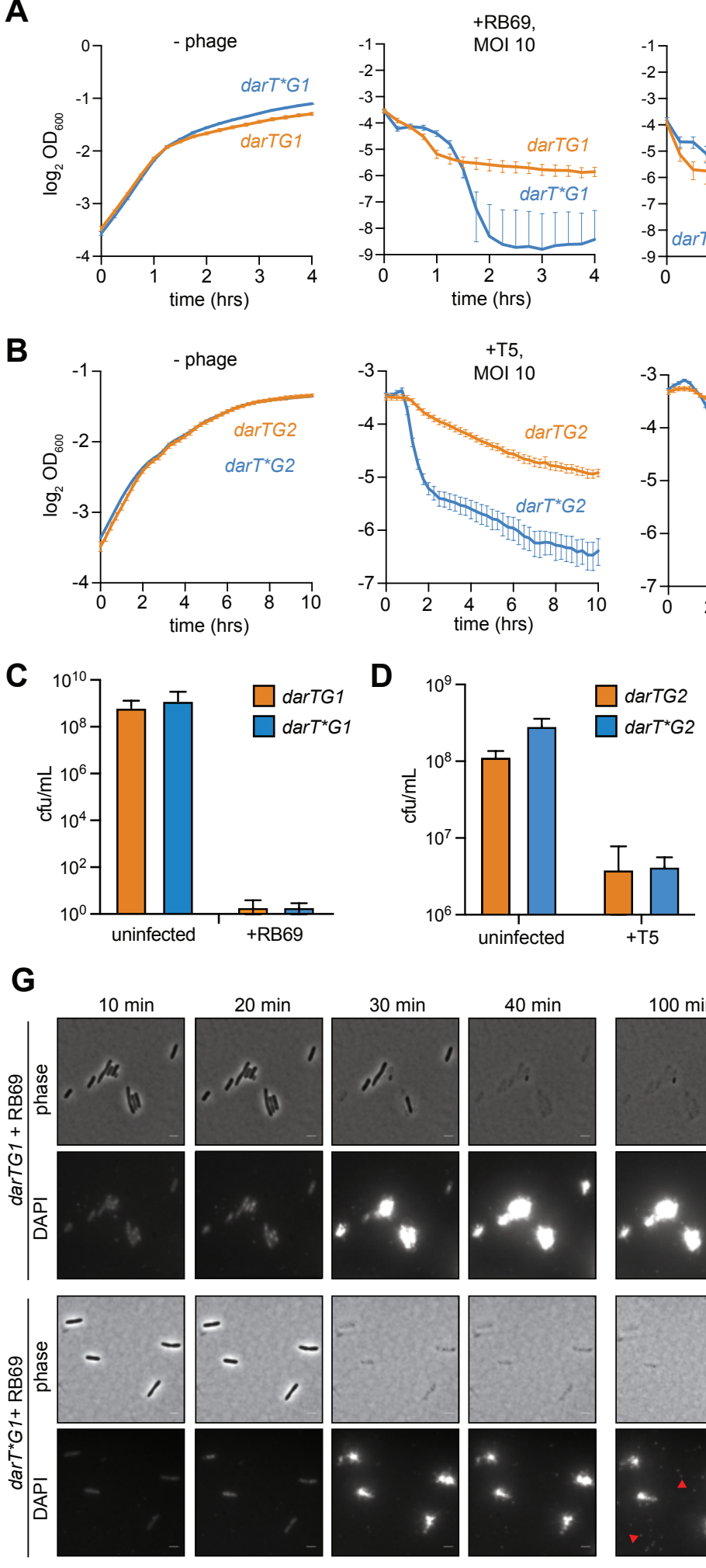
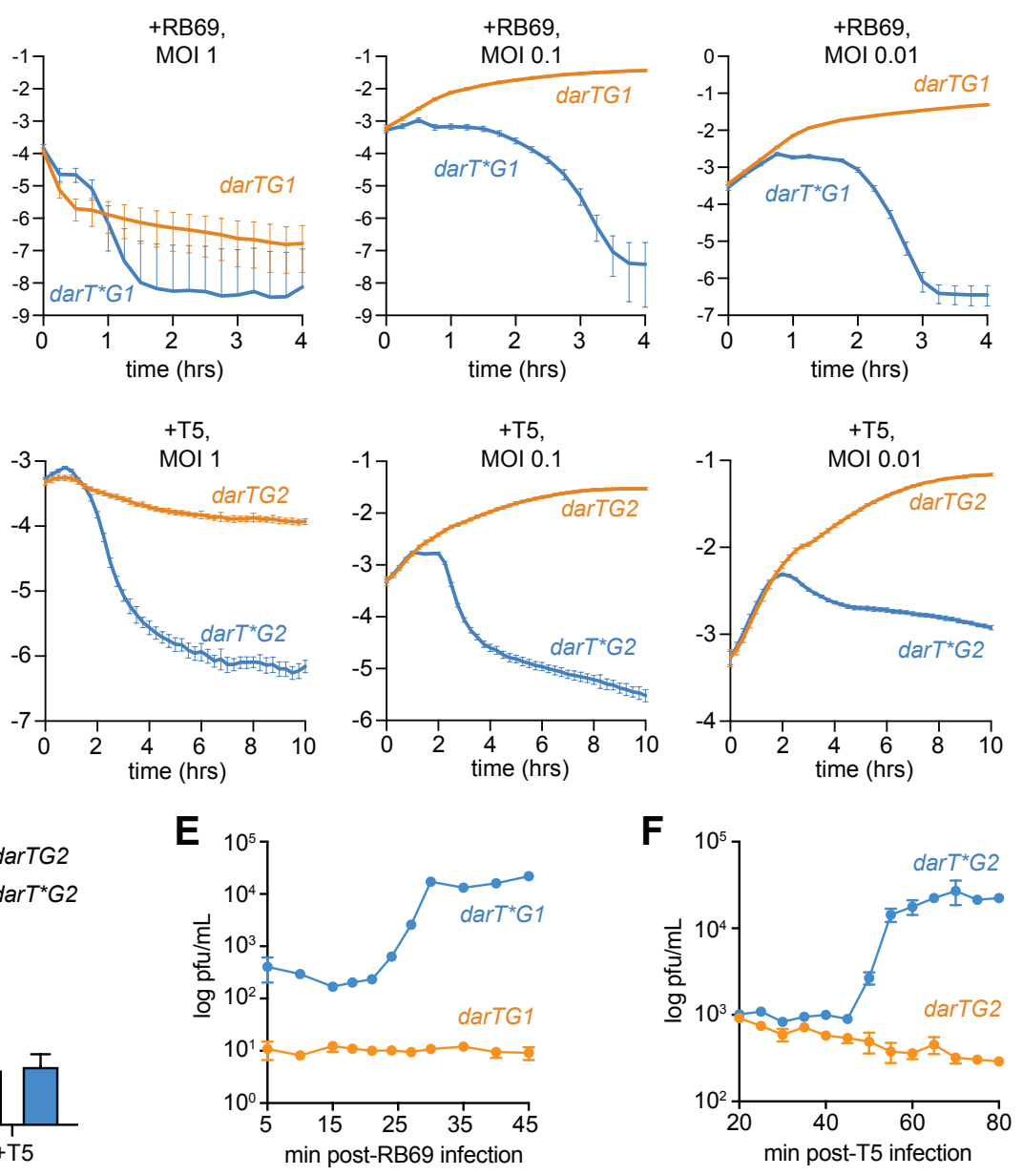

H

$100 \mathrm{~min}$
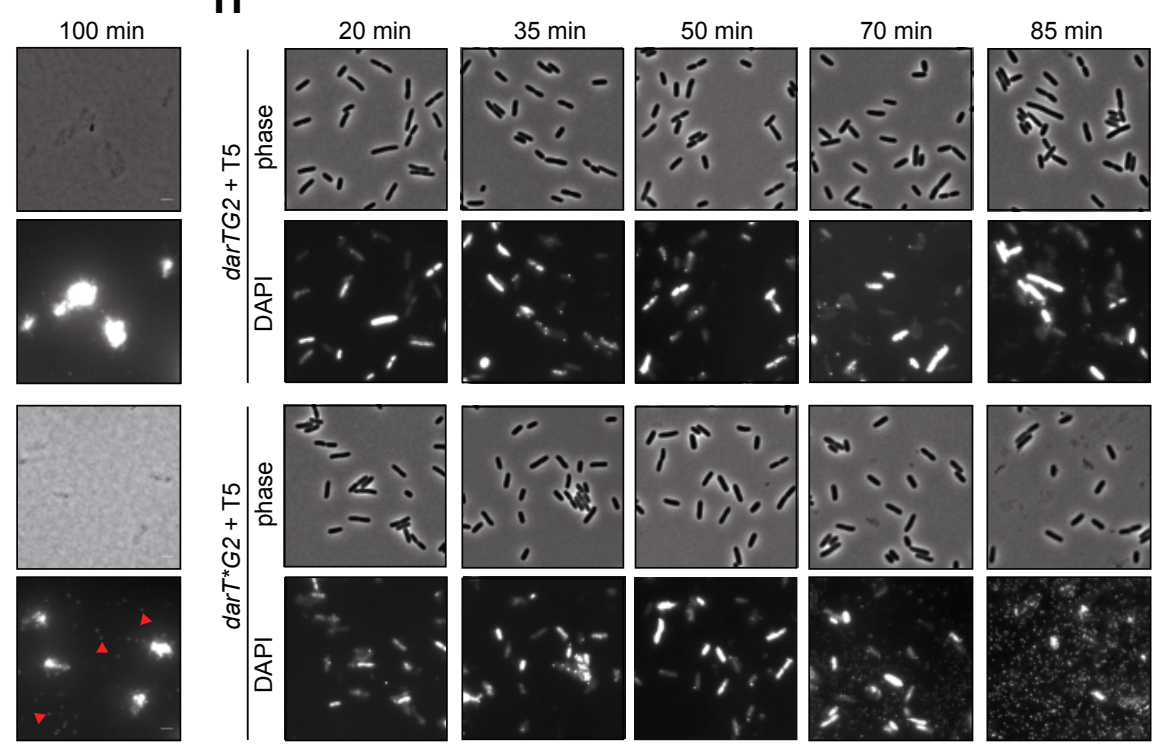

Figure 2. DarTG systems provide phage defense via an abortive infection mechanism.

(A-B) Growth curves for strains with the indicated plasmid-encoded TA system after infection with RB69 (A) or T5 (B) phage at varying multiplicities of infection (MOI).

(C-D) Survival of $E$. coli encoding the indicated DarTG systems as measured by colony forming units (cfu) after 15 min of infection with RB69 at MOI 5 (C) or 30 min of infection with T5 at MOI 20 (D).

(E-F) One-step growth curves, showing measurements of phage concentration (plaque forming units (pfu)/mL) over time in cultures of DarTG1- or DarT ${ }^{*}$ G1 containing cells infected with RB69 (E) or DarTG2- or DarT ${ }^{*} \mathrm{G} 2$ containing cells infected with $\mathrm{T} 5$ (F) at MOI 0.01 during the first round of infection.

(G) Time-lapse microscopy of DAPI-stained RB69-infected E. coli encoding the indicated DarTG1 systems. Red arrowheads indicate puncta corresponding to released viral particles.

(H) Time course microscopy of DAPI-stained T5-infected E. coli bearing plasmids with the indicated DarTG2 systems. 
bioRxiv preprint doi: https://doi.org/10.1101/2021.09.27.462013; this version posted September 27, 2021. The copyright holder for this preprint (which was not certified by peer review) is the author/funder, who has granted bioRxiv a license to display the preprint in perpetuity. It is mage
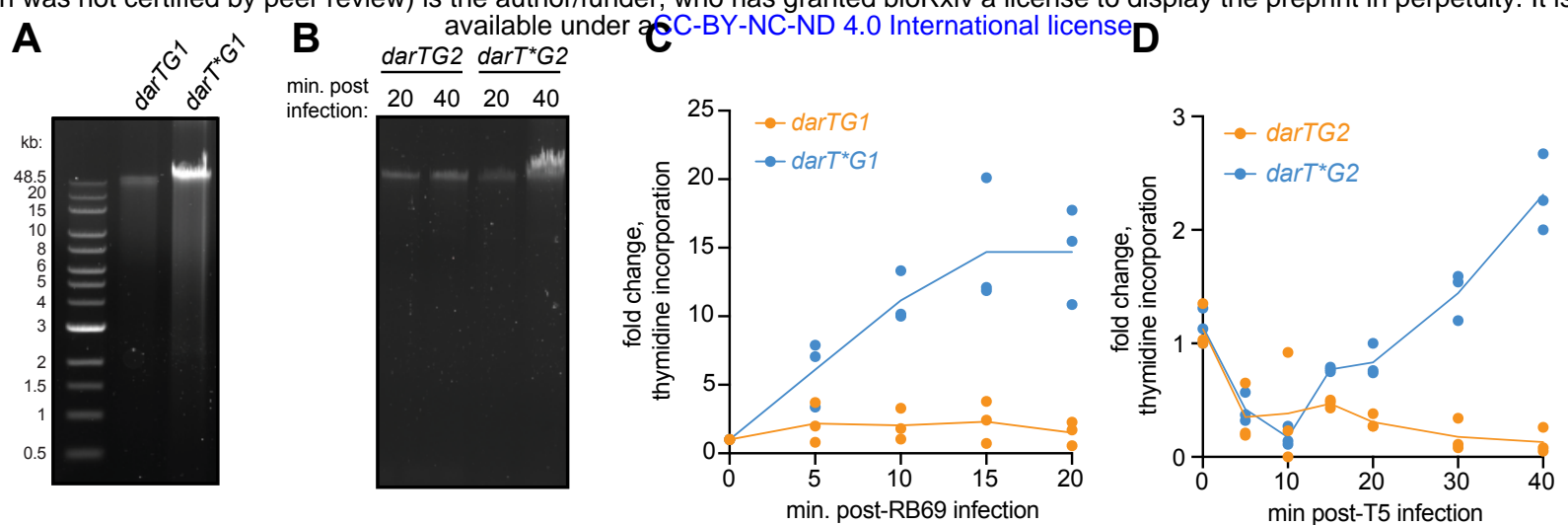

E

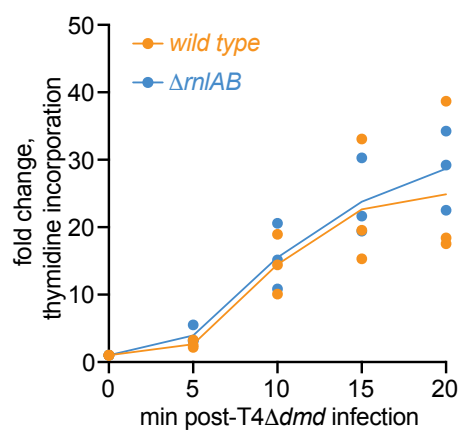

G

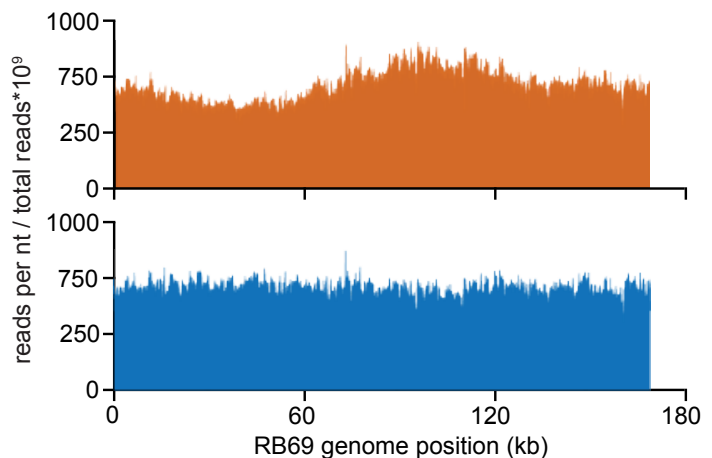

RB69 genome position (kb)

$\mathbf{J}$

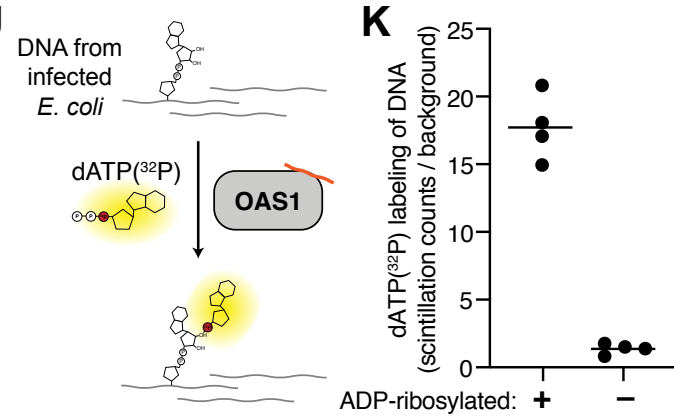

$\mathbf{F}$

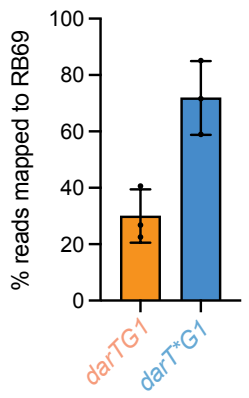

H

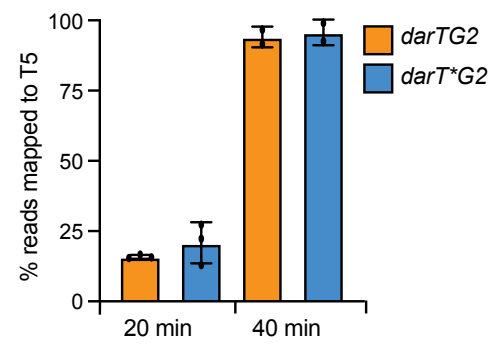

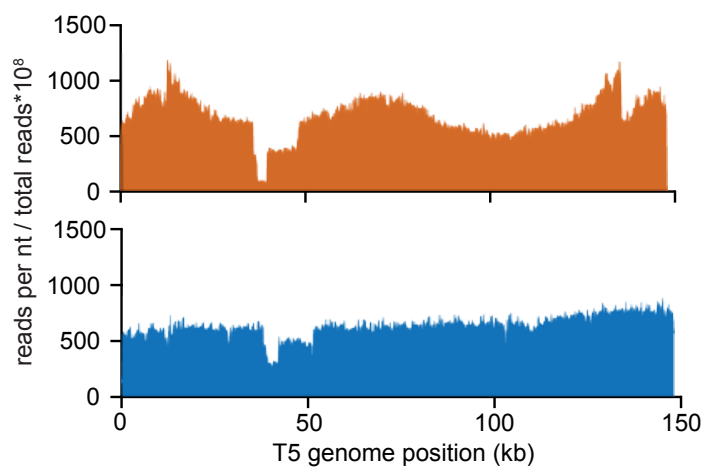
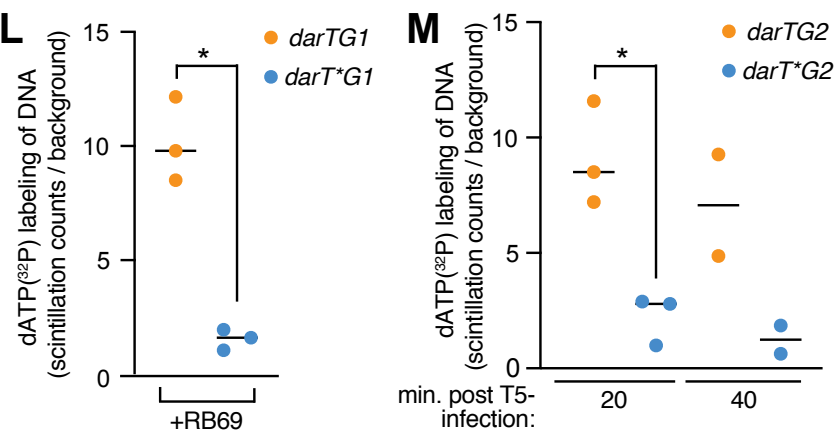

Figure 3. DarTG inhibits phage DNA replication by ADP-ribosylating viral DNA.

(A-B) DNA extracted from equal volumes of $E$. coli cultures harboring the indicated DarTG systems infected with (A) RB69 at MOI 5 at 15 min post-infection or (B) T5 at MOI 20 at 20 and 40 min post-infection and resolved on a $0.5 \%$ agarose gel.

(C-E) DNA synthesis rates as measured by ${ }^{3} \mathrm{H}$-labeled thymidine incorporation at the indicated time points after infection of strains encoding the indicated TA systems infected with RB69 at MOI 5 (C), T5 at MOI 20 (D), or T4 $4 d m d$ at MOI 5 (E).

(F) Fraction of RB69 phage versus host-derived DNA, as determined by Illumina sequencing, for the DNA extracted as in (A).

(G) Sequencing read coverage from experiment in $(F)$.

(H-I) Same as (F-G) but for T5 infection, as in (B).

(J) Overview of an ELTA assay, wherein DNA extracted from infected E. coli cells is incubated with OAS1 protein activated with poly $\mathrm{I}: \mathrm{C}$, and ${ }^{32} \mathrm{P}$-dATP. The resulting stable incorporation of ${ }^{32} \mathrm{P}-\mathrm{dATP}$ onto ADP-ribose modifications is measured by scintillation counting.

(K) ELTA measurements of ADP-ribosylation of DNA of a ssDNA DNA fragment ADP-ribosylated in vitro compared to unribosylated DNA extracted from $E$. coli.

(L-M) ELTA measurements of ADP-ribosylation of DNA from E. coli encoding the indicated DarTG systems after infection with RB69 at MOI 515 min post-infection (K) or T5 at MOI 20 (L) at 20 or 40 min. post-infection. Asterisks indicate $p<0.05$ (t-test). 
A

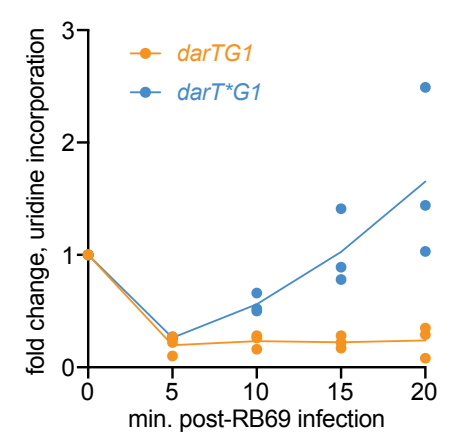

C

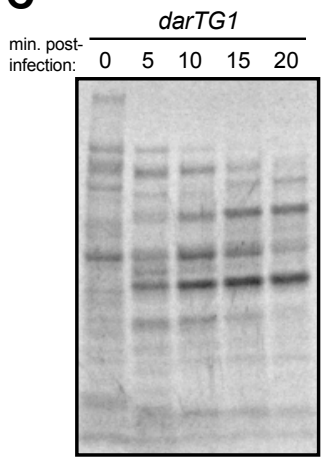

B

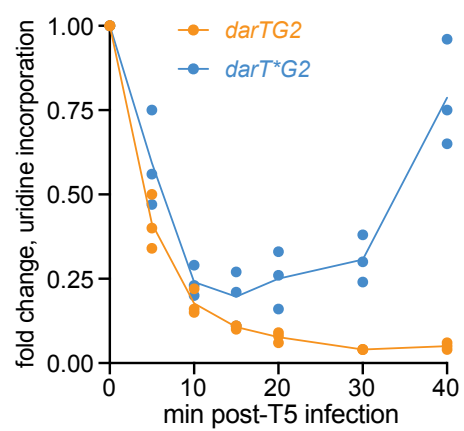

D

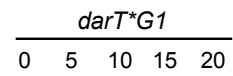
min. post-
infection:

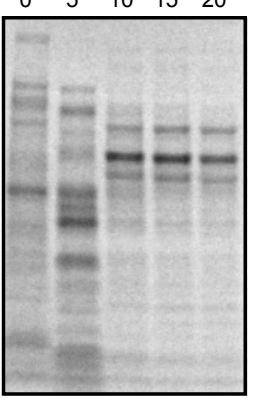

\begin{tabular}{l} 
darTG2 \\
\hline $0 \quad 10 \quad 2030 \quad 40$
\end{tabular}

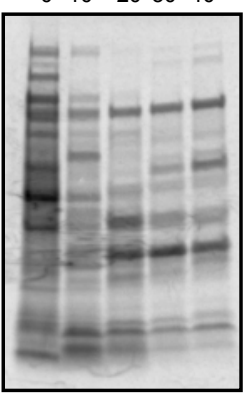

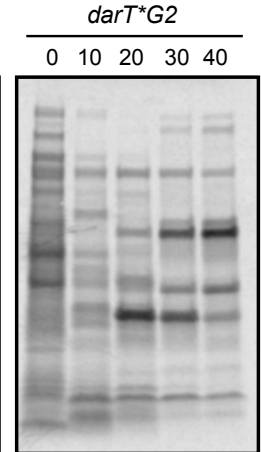

Figure 4. Activated DarT inhibits RNA synthesis and the timing of phage protein production.

(A-B) RNA synthesis rates as measured by ${ }^{3} \mathrm{H}$-labeled uridine incorporation at various time points after infection of strains encoding the indicated TA systems infected with RB69 at MOI 5 (A), T5 at MOI 20 (B).

(C-D) Protein synthesis rates as measured by ${ }^{35}$ S-labeled cysteine and methionine incorporation at various time points after infection for E. coli encoding the indicated TA systems and infected with either RB69 at MOI 5 (D) or T5 at MOI 20 (E) and resolved by SDS-PAGE. 
A

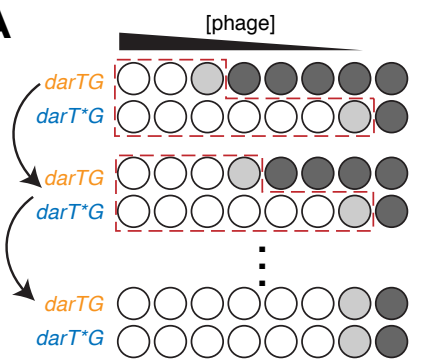

B
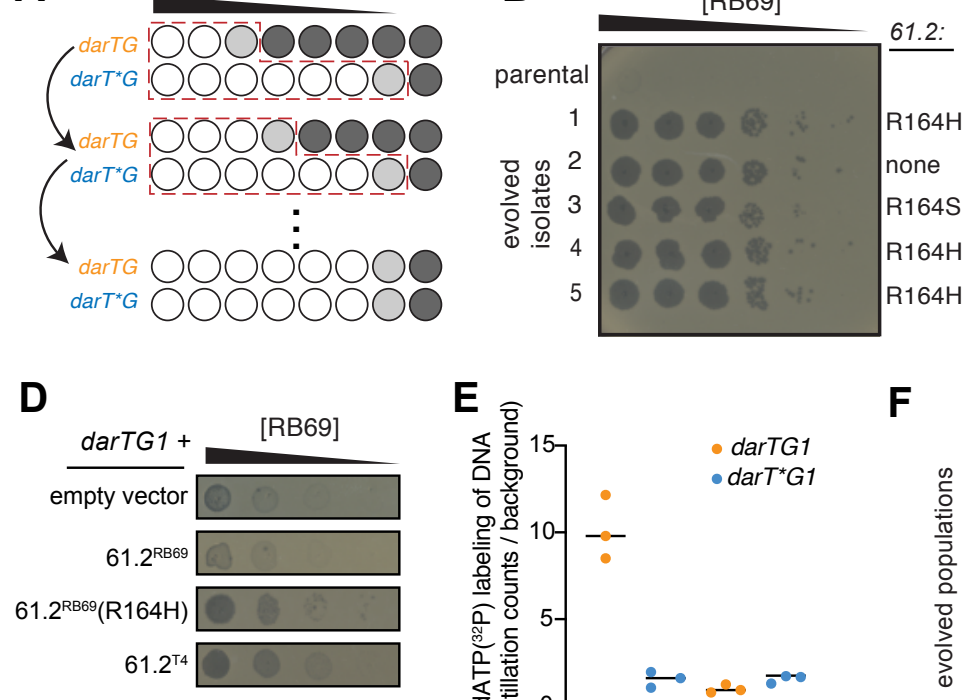

$E_{0}$

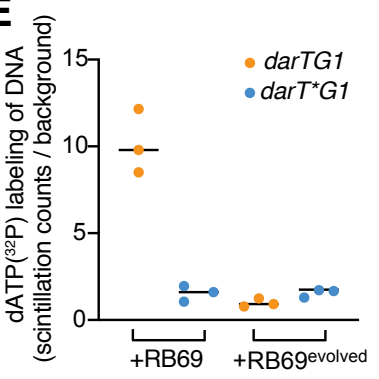

$\mathbf{F}$

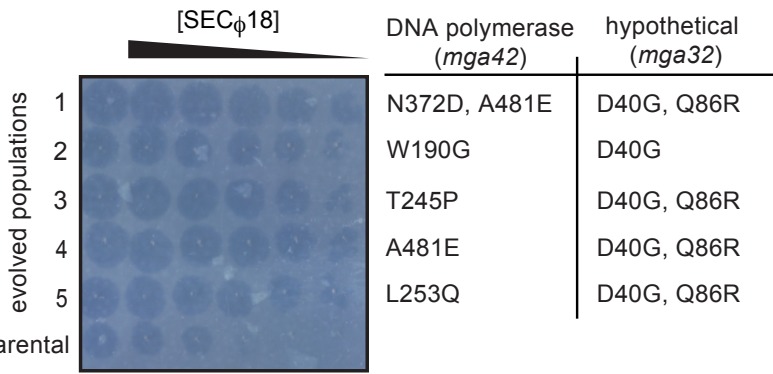

G

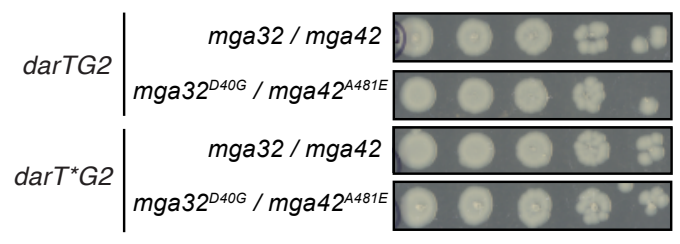

C

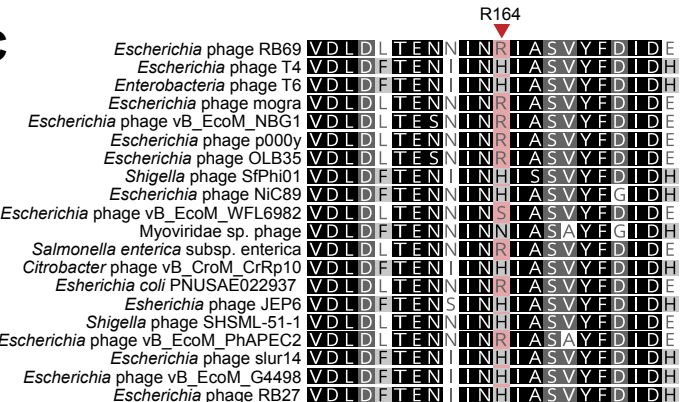

\begin{tabular}{l|l}
\multicolumn{1}{c}{$\begin{array}{c}\text { DNA polymerase } \\
(\text { mga42 })\end{array}$} & \multicolumn{1}{c}{$\begin{array}{c}\text { hypothetical } \\
(\text { mga32 })\end{array}$} \\
\hline N372D, A481E & D40G, Q86R \\
W190G & D40G \\
T245P & D40G, Q86R \\
A481E & D40G, Q86R \\
L253Q & D40G, Q86R
\end{tabular}

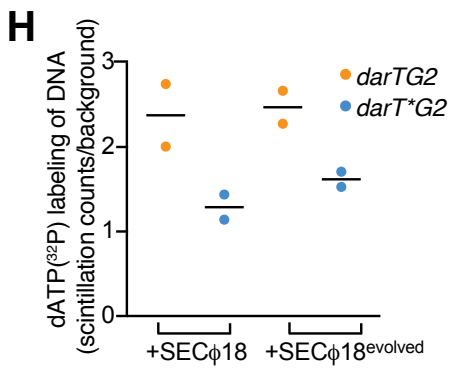

Figure 5. RB69 and SEC $\phi 18$ escape DarTG-mediated defense by two distinct mechanisms.

(A) Overview of phage evolution experiment. DarTG and DarT*G-producing cells seeded in 96-well plates were infected with 10 -fold serial dilutions of phage, with the highest well infected at an $\mathrm{MOI}$ of $\sim 10$. Following incubation, all cleared and partially cleared wells (dashed red lines) were pooled and the resulting phage used to re-infect cells. This process was repeated until phage were able to clear an increasing number of wells of DarTG-containing cells.

(B) EOP of ancestral and evolved RB69 on DarTG1-containing cells. The mutations identified in gene 61.2 following genome sequencing are indicated on the right.

(C) Sequence alignment of the region surrounding residue 164 of gp61.2 for 20 homologs.

(D) EOP of wild-type RB69 on E. coli harboring DarTG1 and either bearing an empty vector or expressing the wild-type RB69 gp61.2, the evolved RB69 gp61.2(R164H), or the wild-type T4 gp61.2.

(E) ELTA-based measurements of ADP-ribosylation of DNA extracted from E. coli encoding darTG1 or darT $T^{\star} G 1$ and infected with an evolved RB69 clone. Data of wild-type RB69 infection (Fig. 3K) is reproduced here for comparison.

(F) Plaque assays showing the increased resistance of five independently evolved populations of SEC $\phi 18$ selected to overcome DarTG2 defense, with the mutations identified by whole-genome sequencing of each population.

(G) Expression of the wild-type and evolved alleles of $m g a 32$ and $m g a 42$ genes in cells containing DarTG2 or DarT*G2.

(H) ELTA-based measurements of ADP-ribosylation of DNA extracted from E. coli encoding darTG2 or darT $T^{\star} G 2$ and infected with the parental SEC $\phi 18$ strains or an evolved SEC $\phi 18$ clone. 
- DarTG
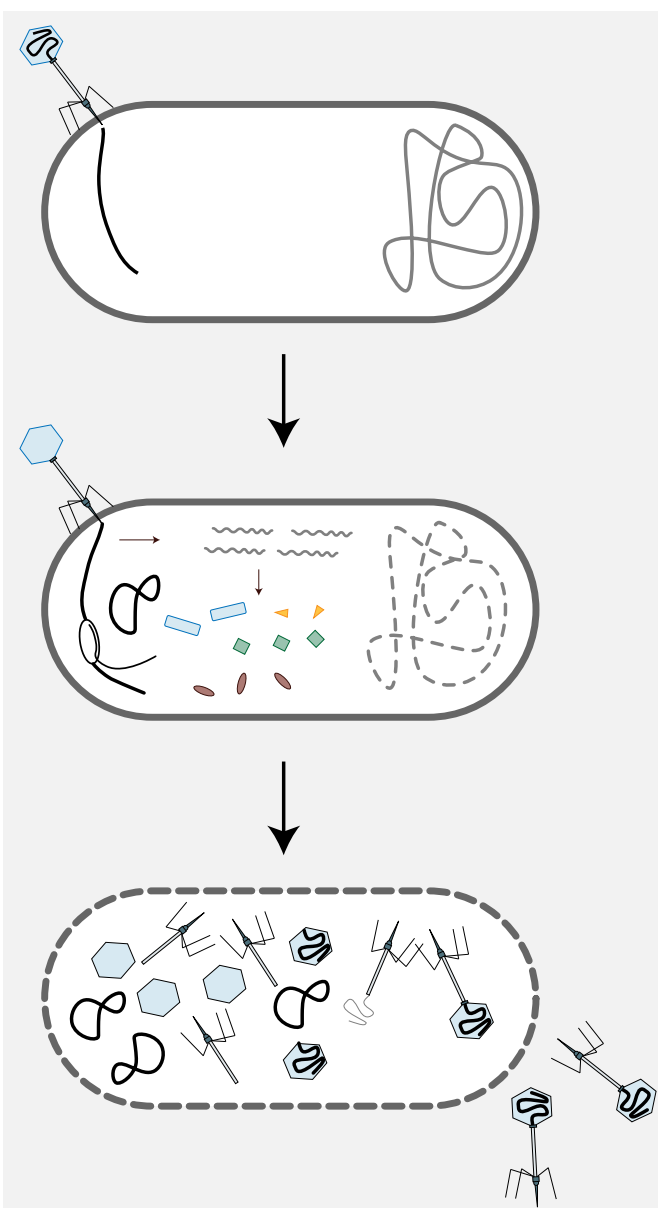

\section{+ DarTG}
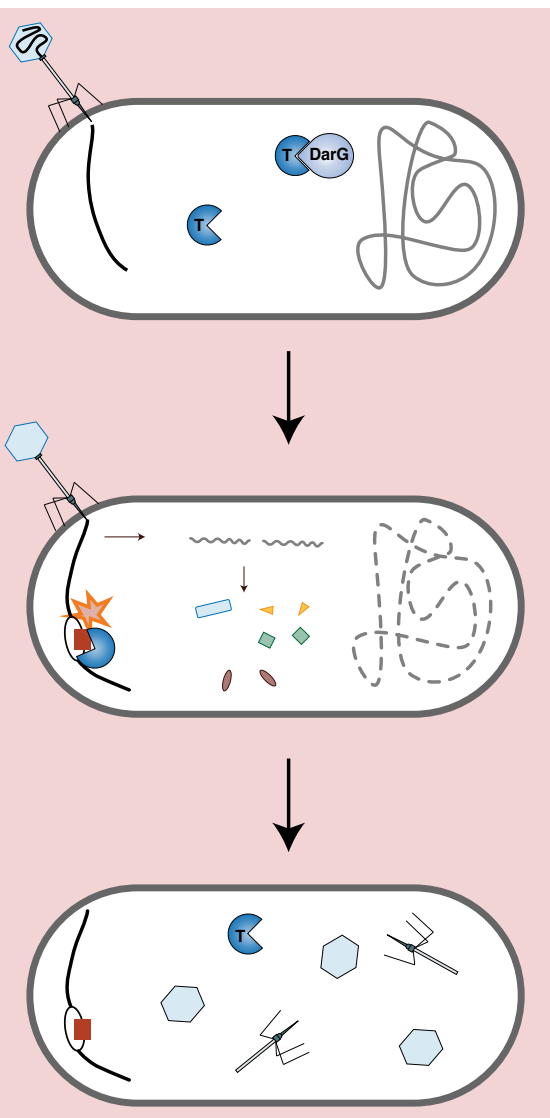

\section{Figure 6. Model for DarTG-mediated defense against phage.}

When DarTG is not present (left), phage adsorb, inject their DNA and begin producing mRNAs and proteins. These proteins enable them to degrade the host chromosome, replicate their DNA, and produce the structural components required to make new phage particles. These components are assembled into capsids into which newly replicated genomes are packaged prior to lysis of the host cell.

When DarTG is present (right), phage infection triggers release of the DarT toxin. The toxin ADP-ribosylates phage DNA, thereby inhibiting DNA synthesis and reducing transcription. Because some transcription occurs, particularly early on, phage are able to degrade the host chromosome and the host cell does not recover. However, due to the absence of newly replicated phage genomes and reduced late protein production, new phage particles are not produced. 
Supplemental Table S1. Bacterial and viral strains used in this study.

Bacterial strains

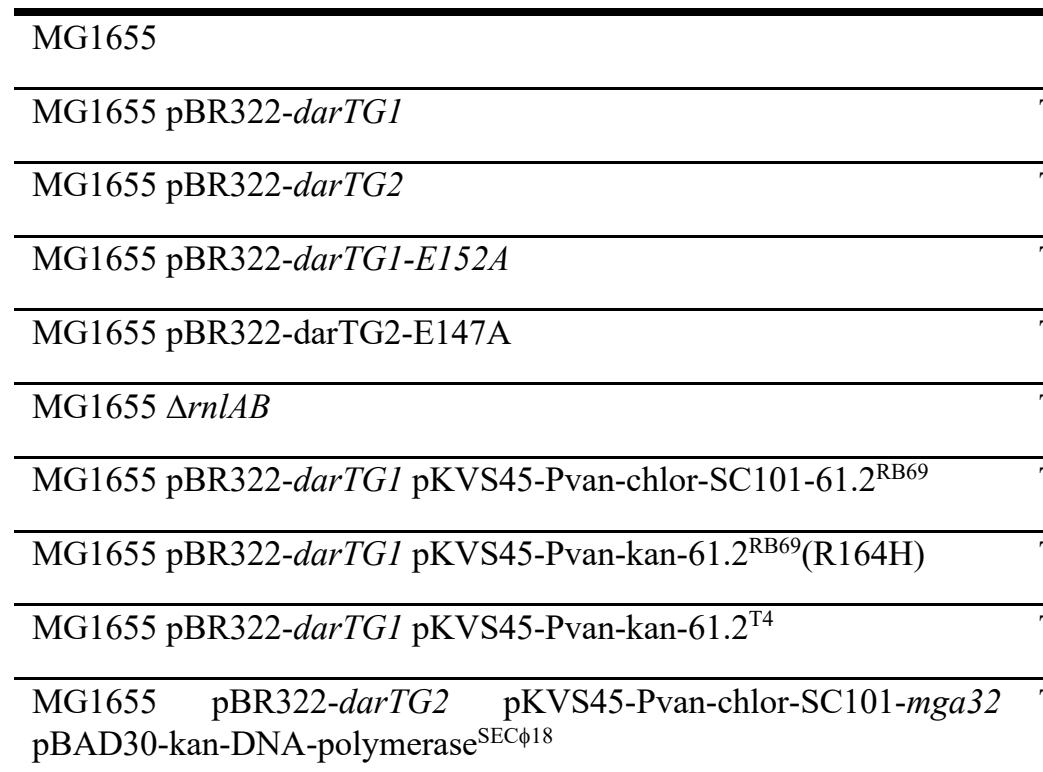

Source This study

This study

This study

This study

This study

This study

This study

This study

This study

pKVS45-Pvan-chlor-SC101- This study

MG1655 pBR322-darTG2 pKVS45-Pvan-chlor-SC
mga32(D40G) pBAD30-kan-DNA-polymerase $\mathrm{e}^{\mathrm{SEC} 18}(\mathrm{~A} 481 \mathrm{E})$
Identifier

ML6

ML3621

ML3622

ML3623

ML3624

ML3625

ML3626

ML3627

ML3628

ML3629

ML3630

ML3631

MG1655 pBR322-darT ${ }^{E 147 A} G 2$ pKVS45-Pvan-chlor-SC101-mga32 This study pBAD30-kan-DNA-polymerase ${ }^{\mathrm{SEC} \phi 18}$

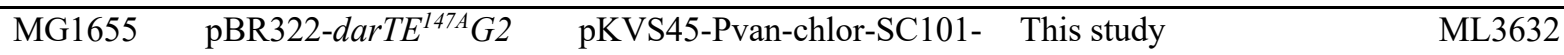
mga32 ${ }^{\mathrm{D} 40 \mathrm{G}}$ pBAD30-kan-DNA-polymerase ${ }^{\mathrm{SEC} \phi 18}(\mathrm{~A} 481 \mathrm{E})$

\section{Phage strains}

\begin{tabular}{lll}
\hline T2 & ATCC Cat \#: 11303-B5 & 3633 \\
\hline T3 & ATCC Cat \#: 11303-B6 & 3634 \\
\hline T4 & Gift from R. Young & 3635 \\
\hline T5 & ATCC Cat \#: 11303-B5 & 3636 \\
\hline T6 & ATCC Cat \#: 11303-B5 & 3637 \\
\hline T7 & Gift from R. Sorek & 3638 \\
\hline RB69 & Laval Collection, HER \# & 3639 \\
& 158 & \\
\hline$\lambda$-vir & Gift from R. Sorek & 3640 \\
\hline SEC $\phi 17$ & (Doron et al., 2018) & 3641 \\
\hline SEC $\phi 18$ & (Doron et al., 2018) & 3642 \\
\hline SEC $\phi 27$ & (Doron et al., 2018) & 3643 \\
\hline
\end{tabular}


bioRxiv preprint doi: https://doi.org/10.1101/2021.09.27.462013; this version posted September 27, 2021. The copyright holder for this preprint (which was not certified by peer review) is the author/funder, who has granted bioRxiv a license to display the preprint in perpetuity. It is made available under aCC-BY-NC-ND 4.0 International license.

\begin{tabular}{lll}
\hline Lust & (Malki et al.) & 3644 \\
\hline T4 $\Delta d m d$ & $\begin{array}{l}\text { (Otsuka and Yonesaki, } \\
\text { 2012) }\end{array}$ & 3645 \\
\hline SEC $\phi 18$ evolved population 1 & This study & 3646 \\
\hline SEC $\phi 18$ evolved population 2 & This study & 3647 \\
\hline SEC $\phi 18$ evolved population 3 & This study & 3648 \\
\hline SEC $\phi 18$ evolved population 4 & This study & 3649 \\
\hline SEC $\phi 18$ evolved population 5 & This study & 3650 \\
\hline SEC $\phi 18$ evolved population 1 clone & This study & 3651 \\
\hline RB69 evolved clone 1 & This study & 3652 \\
\hline RB69 evolved clone 1 & This study & 3653 \\
\hline RB69 evolved clone 1 & This study & 3654 \\
\hline RB69 evolved clone 1 & This study & 3655 \\
\hline RB69 evolved clone 1 & This study & 3656 \\
\hline RB69 evolved clone 1 & This study & 3657
\end{tabular}


Supplemental Table S2. Primers used in this study

Number Purpose Sequence (5'-3')

MLR1 DarTG1 into pBR322 backbone

GCGTATCACGAGGCCCTTTCGTCTTCAAGAATTCTCATGTAT GGTAAATAACTGTAATTTATAGCTTTTATTTGAAGTAGATCA CATGCGTATCCTTTGTGGATACTTGAGGTATATGTTATATAT AAAACATATATTTACATGAAGTAAATGGATGTTTTATGACAA TACAGGAAATAATTCAGCAACGTAATATTCGTAGCTTGTTTC ATTTTACACATAGCGATAATCTAACTTCCATTTTAGATAATG GCCTTATGTCTCGTTCAGAACTTGATAATGAAAATAATGAAT ATAATTGTAATGATGAAGAAAGGATTGATGGTCATCCAGAT GCGATTTGTTTGTCCGTAAGTTACCCTAATGCAAAAATGTTT TATAAATATAGATGTTTAAAGCCTGGGGACTGGGTTATATTA GAAATCAATCCATCTGTTCTTTGGGCTAAGGATTGCGCTTTT TATCCTACCAACGCAGCTTCGAATAACGTTCGTTTTATAAAT CTTGATTTGATGAAAGGAGCTGAGGCATTTAGTGCTTTGTTT TCAGAGAATGTTTTTGGGATTCAGAGAGATGTAAACCTTCCA AGTGAGTATACGACGGATGTTCAAGCTGAAATACTGGTATTT GAAAAAATTCCTCCCTCATATATTATAAGCACTTTTCACCCT AACAAAGAATCTGCTGAACATTTCAAGAGATTATATCCACA GACAATACAGAGATATTATGATAATCTTAATGCTAGAACAC TTTATTCTCAGAGGCATTATTATTTAGGATAAGTTATGGCTG TTAGACCTGTTTTCGTCCCTACAAACGCAGGGAACTTACTAT CGATCACGAAGGATGTGGATTTTCCTTGGGCTCCTGGTATGT CTAAAACGCAAAAGCAAAAATCGATCAGGGCGTTACATACA GCAGCGAATGAGCAGGGATTGAACTCTTTGTTGGAAATTTCT AGTAAATCCGAAGATGCGCTGGGGGTAGCACTTAGTGCATT TAATCTAAGAATAAAGACGAAAAGACTTGGGAAAGAGTTTA CAGTTGAATCTGCATTTCAAGCAAGTAAAGTATTTGAAATGG GAGGACCATATGTCGATATCTTAGATAAAAGCTCGATAGAA GCAAAAAAAGATATGAGATTAAAGGAATCTGGGGGATTAGT TAACTTCAAATTTTATAATACAATATGGCCCATTGTCCCCAG GACTGCTTTTTATGATTGGTTATATTTGAGTGCGCTCAATCA AAACAAAAATCTAGCACTCCATCTATTAAATTTTGATGGGTT TACTGATATTGAATTTAATCCAGCGAAATCTATTAATTGCCA AGCCAGAGCAGCTGCACTTTTTGTCTCTTTAGTAAGACGAAA TATGTTAGATGATGTTTTATCTTCAAAGGATGGTTTTTTATCA AAATTAGCATCTCATTATGGTGTTGAAAATTATTCTATTCAA CATACATTGATATGAATAATGGCCTGTAAAGGCCATTTTTAT CTAAAGGCACCTCGCTAACGGATTCACCACTCCAAGAATTG GAGC
MLR2 DarTG2 into pBR322 backbone
GCGTATCACGAGGCCCTTTCGTCTTCAAGAATTCTCATGTCA
AAAAATCGAAAATAATTAGATCGACAGGCAGAAGGCAATGT
GAATAATCTTAAAAACCTTGACTGCCAGTGAGGTTTGGTTCT
ATAAATAGACTTACGAATTTGAAAGGATGAGTTGAAATGAG
CGAAAGGAAAAGAATTGAAGATCAGTATCTTCTCTATCATTT
AACATGTATGGATAATCTACCTAGTATTTTAGACACAGGATT
AAGATCTCGTGCATCAGTAAAAGGTGAGTTTGTTGATGTGGC
CGACGGTGAAATAATAACAGGTCGCGAAGCATTAAATTTGC
AAACAATGGTTCCTTTTCATTTCTTTACTAAGAATCCATTTGA
TGGAAGAGTCCTGAAAGATCATAAGGAAAAATCATTTTGTA
TTATTTCGGTTTGTCGGGCATTTGCCAAAGAAAATGGTTGGA
AAGTTATCCCTAAACATCCATTATCCACCAGTTCTGTTATAG
ACCTCTTAGATTATGAACAAGGAATGGCCCAAATTGACTGG

32 
GAGCTTATGAATATAAGGGATTATAAAAATGCAGAATGCAA ATCTGTATGTATGGCTGAGTGTTTATCAAGTGTAACGGTCGA GCCGAAAAATTTCCATTGCATCTTTGTCAAAAATGATAACGA AAAAAAATATGTTGAAGGGTTGATAAAACAACAAGGATTAA GTATATTGATAACGATCAACACTTATATGTTCTAATTCTATG ATTAAGTATGCAAGCGGAAATCTTCTAAACTCTACATCGCAA GCTCTTGTGAATGCTGTAAACTGCCAAGGAGTCATGGGAAA GGGTATTGCTTTGGCTTTTAAAGAAACTTTTCCCTATAATTTC GAAGTTTATAAGAGAGCATGCACCACTGGTACAATGAAAAT TGGACAGGTTTTAATTGTAGAGGAAAAGGGAAAAATAATCG TAAACTTTCCCACTAAAGATAGTTGGAGAAAAAAATCAACA TATGATTTTATCTCGCAAGGCCTTGAATCTTTAGCAAAAATT ATAGACGAAAAAAAAATTACATCAATATCAATACCACCTTT AGGCTGTGGTAATGGGGGCCTCGATTGGAATAAGGTTGAGG CTCTTATTTTAAAAACTTTTCAAAATTTAGATAATGTTGATGT GGTAATTTATCCGCCTGCAACTAACAATCAGCTTTCAAAAAA TAAAAATGTTATTAATGCTAAGCATCTTTTGGTTCACTACGC CTATGGAAAATTAAAAGTTAAGCAAAAATATTCCCTTTATAC AGTTTTTTATATTTGCGAATGTATTGAGAAAGCAAATCTTTT TTTATTCGATTTTAAACATGGTCGGCCATACTCTTCTGAGTTA GAAAATATTATGAAGGATATAGCAAACCTTAAAGTTGAGTT GCAAGGAGATTTTGATGCTTTTATTGAAGATTATATTAATAC TCATCAATCCAAAGAGTTACAAACCGAGTTTGGTAAACTAA TCTCAACTTTAAATCCAAGTATAAGTTTTCTCAATGCTCTTA AAAATAAGATTGATTATACAGATAGCGTACATGTGATCACG AGAATTGCTAAAGAAAAAGATCATGGCTTGCATATTGACAA TTTCAAAGAATATGAAAATATTGTGCAGCATTTAATTAAAAA TGGATTGGTAATCGAAGATATTTTTAACGAACTAAAAATAA AGGGAAGTTAGTAGCGCTATTTTCAATTTTTTTGCTGAGGCA CCTCGCTAACGGATTCACCACTCCAAGAATTGGAGC

\begin{tabular}{lll}
\hline MLR3 & Linearize pBR322 & GGCACCTCGCTAACGGAT \\
\hline MLR4 & Linearize pBR322 & ACATGAGAATTCTTGAAGACGAAAGG \\
\hline MLR5 & $\begin{array}{l}\text { Generate E152A substitution in } \\
\text { DarT1 }\end{array}$ & ATACTGGTATTTGAAAAAATTCCTCCCTC \\
& Generate E152A substitution in & CGCAGCTTGAACATCCGTCGTATAC \\
& DarT1 & \\
\hline MLR6 & Generate E147A substitution in & GCTGCCTGTTTATCAAGTGTAACGGTCGAG \\
& DarT2 & \\
\hline MLR8 & Generate E147A substitution in & CATACATACAGATTTGCATTCTGCAT \\
& DarT2 & \\
\hline MLR9 & $\begin{array}{l}\text { MGA32 from SECf18 into } \\
\text { pKVS45-Pvan-Cm-Sc101 }\end{array}$ & $\begin{array}{l}\text { ACAGCCTCTACAAATAATTTTGTTTAAATTAAAGAGGAGAA } \\
\text { AATGGCAAACTTCGGAGCATT }\end{array}$ \\
\hline MLR10 & $\begin{array}{l}\text { MGA32 from SECf18 into } \\
\text { pKVS45-Pvan-Cm-Sc101 }\end{array}$ & GAGGCCTCTTTTCTGGAATTTGGTACCGAGTCATATCCCATC \\
& CACGTTAATTGCG \\
\hline MLR11 & Linearize pKVS45-Pvan_Cm- & CTCGGTACCAAATTCCAGAAAAGAG \\
& SC101_linearize
\end{tabular}


MLR12 Linearize pKVS45-Pvan_CmSC101_linearize

\begin{tabular}{|c|c|c|}
\hline MLR13 & $\begin{array}{l}\text { DNA polymerase from SECf18 } \\
\text { into pBAD30-kan }\end{array}$ & $\begin{array}{l}\text { TCCATACCCGTTTTTTTGGGCTAGCGAATTATTAAAGAGGAG } \\
\text { AAAATGAAAGACTTCGAACGTCTGTTT }\end{array}$ \\
\hline MLR14 & $\begin{array}{l}\text { DNA polymerase from SECf18 } \\
\text { into pBAD30-kan }\end{array}$ & $\begin{array}{l}\text { CTTCTCTCATCCGCCAAAACAGCCAAGCTTTTACTTGCGGTA } \\
\text { TCGATACATACAATC }\end{array}$ \\
\hline MLR15 & Linearize pBAD30 & AAGCTTGGCTGTTTTGGC \\
\hline MLR16 & Linearize pBAD30 & AATTCGCTAGCCCAAAAAAACG \\
\hline MLR17 & $\begin{array}{l}61.2 \text { from RB69 into pKVS45- } \\
\text { Pvan-Kan }\end{array}$ & $\begin{array}{l}\text { AGCTCAGTCCTAGGTACCATTGGATCCAATTGAATAATATGA } \\
\text { TTTATTACATGCACAAAAACG }\end{array}$ \\
\hline MLR18 & $\begin{array}{l}\text { 61.2 from RB69 into pKVS45- } \\
\text { Pvan-Kan }\end{array}$ & $\begin{array}{l}\text { GAGGCCTCTTTTCTGGAATTTGGTACCGAGTTAACCTCGATT } \\
\text { CATAAATGCATTAAATATTTGG }\end{array}$ \\
\hline MLR19 & $\begin{array}{l}61.2 \text { from T4 into pKVS45-Pvan- } \\
\text { Kan }\end{array}$ & $\begin{array}{l}\text { AGCTCAGTCCTAGGTACCATTGGATCCAATCGCTGAAGTGGT } \\
\text { AGGTGAATAATGCTTTATC }\end{array}$ \\
\hline MLR20 & $\begin{array}{l}61.2 \text { from T4 into pKVS45-Pvan- } \\
\text { Kan }\end{array}$ & $\begin{array}{l}\text { GAGGCCTCTTTTCTGGAATTTGGTACCGAGTTAACCTCTATT } \\
\text { CATAAAAGCATTAAAAATTTGGTCAT }\end{array}$ \\
\hline MLR21 & Linearize pKVS45_Pvan_kan & ATTGGATCCAATGGTACCTAGGACTG \\
\hline MLR22 & $\begin{array}{l}\text { Amplify sacB-neo cassette with } \\
\text { ends flanking } r n l A B\end{array}$ & $\begin{array}{l}\text { GGGGTATTATTGTAGAGTTTCCCCATATGTTTCTATGGGATC } \\
\text { CAGGAACCTTTTATGATTTTCTATCAAAC }\end{array}$ \\
\hline MLR23 & $\begin{array}{l}\text { Amplify sacB-neo cassette with } \\
\text { ends flanking } r n l A B\end{array}$ & $\begin{array}{l}\text { CATGCCAAAAGGGCGAATTCTATACTGGTTCGTTTAGAAAG } \\
\text { AAGAGTTCTTGGTCGGTCATTTCGAAC }\end{array}$ \\
\hline MLR24 & $\begin{array}{l}\text { RnlAB deletion oligo } \\
*=\text { phosphothioation }\end{array}$ & $\begin{array}{l}\text { G*A*ATTCTATACTGGTTCGTTTAGAAAGAAGAGTTTCAAAA } \\
\text { TGTCATTTCCTGGATCCCATAGAAACATATGGGGAAACT }\end{array}$ \\
\hline MLR25 & $\begin{array}{l}\text { Oligo used in control ADP- } \\
\text { ribosylation reaction }\end{array}$ & $\begin{array}{l}\text { TCGACGATCATGCGACTGAGCTCGATTCGAGTCATATGCTAG } \\
\text { GGCCAGACCTTTGAGCTGTACAAGTCAGATCTCGAGCTCCA } \\
\text { ACGCGCTTCGAGTATGTATTGGCCAATACGTGGGCTTAGTCC } \\
\text { ACGCCATTGATTGGAACTAGGGGCTACCTTGCTCACAGATCT } \\
\text { GCGGTTACGAAATACTCCGCTG }\end{array}$ \\
\hline
\end{tabular}

